\title{
Improving the Performance of the Zero- Forcing Multiuser MISO Downlink Precoder Through User Grouping
}

\author{
Saif Khan Mohammed and Erik G Larsson
}

\section{Linköping University Post Print}

\section{Tweet}

N.B.: When citing this work, cite the original article.

(C2016 IEEE. Personal use of this material is permitted. However, permission to reprint/republish this material for advertising or promotional purposes or for creating new collective works for resale or redistribution to servers or lists, or to reuse any copyrighted component of this work in other works must be obtained from the IEEE.

Saif Khan Mohammed and Erik G Larsson, Improving the Performance of the Zero-Forcing Multiuser MISO Downlink Precoder Through User Grouping, 2016, IEEE Transactions on Wireless Communications, (15), 2, 811-826.

http://dx.doi.org/10.1109/TWC.2015.2478878

Postprint available at: Linköping University Electronic Press

http://urn.kb.se/resolve?urn=urn:nbn:se:liu:diva-126260 


\title{
Improving the Performance of the Zero-Forcing Multiuser MISO Downlink Precoder through User Grouping
}

\author{
Saif Khan Mohammed, Member, IEEE, and Erik G. Larsson, Senior Member, IEEE
}

\begin{abstract}
We consider the Multiple Input Single Output (MISO) Gaussian Broadcast channel with $N_{t}$ antennas at the base station (BS) and $N_{u}$ single-antenna users in the downlink. We propose a novel user grouping precoder which improves the sum rate performance of the Zero-Forcing $(\mathrm{ZF})$ precoder specially when the channel is ill-conditioned. The proposed precoder partitions all the users into small groups of equal size. Downlink beamforming is then done in such a way that, at each user's receiver the interference from the signal intended for users not in its group is nulled out. Intra-group interference still remains, and is cancelled through successive interference pre-subtraction at the BS using Dirty Paper Coding (DPC). The proposed user grouping method is different from user selection, since it is a method for precoding of information to the selected (scheduled) users, and not for selecting which users are to be scheduled. The proposed precoder is a generalization of two special cases, one where each group has only one user (ZF precoder) and another where all users are in a single group (ZF-DP precoder). A larger group size helps in improving the sum rate performance but at the cost of greater complexity. The proposed generalization therefore allows for trade-off between performance and complexity.
\end{abstract}

Index Terms-MIMO broadcast channel, precoding, lowcomplexity, user grouping, dirty paper coding, zero-forcing.

\section{INTRODUCTION}

Multiple-Input Multiple-Output (MIMO) technology holds the key to very high throughput downlink communication in fading wireless channels by exploiting the spatial dimension [1]. However most modern MIMO wireless communication standards support a maximum achievable spectral efficiency of around $10 \mathrm{bits} / \mathrm{sec} / \mathrm{Hz}$. This is partly due to the use of sub-optimal orthogonal multiple access schemes like TDMA and FDMA. The capacity region and sum capacity of the Gaussian MIMO broadcast channel (which models downlink communication in modern wireless systems) is achieved by a scheme called Dirty Paper Coding (DPC), in which all users share the same frequency-time resource [2]. It is also known that orthogonal access schemes (like TDMA, FDMA) are strictly sub-optimal and achieve only a small fraction of the total sum capacity [3]. However, TDMA and FDMA are

S. K. Mohammed is with the Department of Electrical Engineering, Indian Institute of Technology (I.I.T.) Delhi, India and is also associated with the Bharti school of Telecommunication Technology and Management (BSTTM), I.I.T., Delhi. E. G. Larsson is with the Communication Systems Division, Dept. of Electrical Engineering (ISY), Linköping University, 58183 Linköping, Sweden. E-mail: saifkmohammed@gmail.com and erik.larsson@isy.liu.se. The work of Saif Khan Mohammed was partly supported by the EMR funding from the Science and Engineering Research Board (SERB), Department of Science and Technology (DST), Government of India. The work of E. G. Larsson was supported by the Swedish research council and ELLIIT. This paper is a substantial extension of our conference paper presented at IEEE Globecom 2011 [18]. still favored in practice due to the high precoding complexity of optimal precoders like DPC. Apart from DPC, other nearoptimal precoders like those based on vector perturbation and lattice reduction [5], [6] also have prohibitive complexity. On the other hand low complexity precoders, like ZF [7], MMSE are known to achieve poor sum rate performance especially when the condition number of the channel gain matrix is large. ${ }^{1}$ In this paper, a channel realization is said to be illconditioned if the condition number of the channel gain matrix is large, i.e., the channel gain vectors are "nearly linearly dependent" (the channel gain matrix might still have full rank and is not necessarily rank deficient) so that the instantaneous sum rate of the $\mathrm{ZF}$ precoder for that channel realization is significantly less than its ergodic (average) sum rate.

To keep the low-complexity benefit of the ZF precoder and yet improve the overall sum rate (specially when the channel is ill-conditioned), we propose a user grouping based precoder. In the proposed precoder, the users are divided into small groups of equal size. Downlink beamforming is done in such a way that, at each receiver the interference from the signal intended for users not in its group is nulled out. However, there still remains interference from the signal of users in the same group. This interference is pre-cancelled at the transmitter, by performing dirty paper coding among the users in the same group. With small groups (e.g., having only two users), dirty paper coding within each group is practically feasible [8], [9], [10]. Note that the proposed user grouping method is fundamentally different from user selection. User selection schemes select a subset of users to be scheduled [11], [12], [13], [14], [15]. The base station (BS) then precodes information only to these selected users. The proposed user grouping precoder is a method for precoding of information to the selected users, and not for selecting which users are to be scheduled. ${ }^{2}$ Note that the user grouping precoder proposed by us in this paper could be used to significantly improve the overall information sum rate performance achieved by user selection methods which assume a ZF precoder at the BS (for

\footnotetext{
${ }^{1}$ Condition number of a matrix is the ratio of the greatest to the smallest singular value of the matrix.

${ }^{2}$ The proposed user grouping precoder is based on spatial channel sharing between scheduled users, as opposed to temporal channel sharing in user selection schemes. This distinction is the same as that between the work in [16] and that in [13]. In [16] the authors propose a block diagonalization method for precoding of information to already selected users, whereas in [13] the authors propose a method to find the subset of users to be scheduled so that the information sum rate (using a block diagonalization precoder) is maximized.
} 
example the user selection method proposed in [11]).

Inter-group interference pre-cancellation for a group of users is achieved by choosing their beamforming vectors to lie in a space orthogonal to the space spanned by the channel vectors of the users in the other groups. One novel aspect of the proposed precoder is that we choose the beamforming vectors in such a way that the effective channel matrix for each group is lower triangular, which enables successive interference precancellation within each group using DPC. With a group size greater than one, the proposed precoder is analytically shown to achieve a sum rate greater than that achieved by the ZF precoder. For a given grouping of users, the optimal power allocation is given by the waterfilling scheme. Since the achievable sum rate of the proposed precoder is observed to be sensitive towards the chosen grouping of users, the information sum rate is jointly optimized w.r.t. both the per user power allocation as well as the grouping. This optimization problem is inherently complex, and therefore we propose a near-optimal solution to it, which we refer to as JPAUGA (Joint Power Allocation and User Grouping Algorithm).

Through simulations, we show that in ill-conditioned channels the proposed precoder with JPAUGA user grouping achieves a sum rate significantly greater than that achieved by the ZF precoder. Further for the special case of user pairing (i.e., two users in each group), interference pre-cancellation needs to be performed for only one user in each group, for which practical and near-optimal performance achieving (i.e., close to DPC) methods have been reported [8]. Further, with user pairing the complexity of the proposed precoder with JPAUGA user grouping is shown to have a complexity of $O\left(N_{u}^{2} N_{t}\right)$ which is the same as the complexity of the $\mathrm{ZF}$ precoder. A special case of the proposed precoder is when there is only one group containing all the $N_{u}$ users. This special case has been proposed as the ZF-DP precoder in [20]. Though the ZF-DP precoder achieves better performance than the proposed user grouping precoder (with more than one group), it has a much higher complexity. Another special case of the proposed precoder is when there are $N_{u}$ groups with each user being a separate group. Interestingly, this special case is nothing but the low complexity ZF precoder. ${ }^{3}$ Grouping more number of users together (i.e., a larger group size) helps in improving the sum rate performance but at the cost of greater complexity. The proposed user grouping precoder is therefore a generalization of both these special cases, which allows for trade-off between performance and complexity.

We also clarify that, the proposed precoder is entirely different from the block diagonalization based precoder proposed in [16], which considers a MIMO broadcast channel, in which each user could have multiple receive antennas. Beamforming vectors are chosen such that each user sees no interference from the information intended for other users. Hence, in the special case of MISO broadcast channel (which we consider in this paper), the block diagonalization precoder in [16] reduces to the $\mathrm{ZF}$ precoder. In addition to this, the precoder that we propose performs beamforming in groups of users and not

\footnotetext{
${ }^{3}$ With each user as a different group, there is no intra-group interference and beamforming to pre-cancel inter-group interference is nothing but the ZF precoder.
}

separately for each user.

The proposed user grouping precoder is also different from the vector perturbation based user grouping precoder proposed in [17]. In [17], the authors propose to group users with similar modulation alphabets so that vector perturbation could be performed jointly within a group of users in order to reduce intra-group interference. This grouping however does not necessarily optimize the information rates to users, whereas in our paper we explicitly maximize the information sum rate with respect to the user grouping.

The specific contributions of this paper are: i) we propose a novel user grouping based precoder for the Gaussian MISO broadcast channel where inter-group interference is pre-cancelled using beamforming and intra-group interference is cancelled using successive DPC, ii) we propose a joint power allocation and user grouping algorithm (JPAUGA) for optimizing the information sum rate, iii) with two users per group the complexity of the proposed user grouping precoder is the same as that of the $\mathrm{ZF}$ precoder (i.e., $\mathrm{O}\left(N_{u}^{2} N_{t}\right)$ ), iv) with two users per group simulations suggest that the proposed precoder (with JPAUGA user grouping) achieves a significantly larger information sum rate than the ZF precoder, v) exhaustive numerical studies done by us reveal that the proposed user grouping precoder also reduces the probability of the achievable information sum rate being small (i.e., it reduces outages ${ }^{4}$ ).

This paper is a substantial extension of our conference paper [18]. The major additions in this paper are: i) the power allocation in the conference paper was chosen to be that of the ZF precoder whereas in this paper we present the JPAUGA algorithm which starts with the ZF power allocation and improves upon both the user grouping and the power allocation iteratively (see Section V), ii) in the conference paper only the ergodic sum rate performance of different user groupings was compared, and the effect of the chosen user grouping on the distribution of sum rate was not clear, iii) exhaustive simulations in the current paper reveal that even though the ergodic sum rate performance of random user grouping is close to that of the proposed user grouping (based on JPAUGA), outages are more probable when random user grouping is used (see Fig. 4 in Section VI), iv) the fact that the user grouping based precoder achieves a higher sum rate than the $\mathrm{ZF}$ precoder was simply stated in the conference paper and is now rigorously proven in this paper (Appendix A), v) the detailed complexity analysis of the proposed precoder is provided only in the current paper (see Section V-B and Appendix C). Additionally, the exposition of the proposed user grouping precoder is more comprehensive in this paper due to more illustrations, examples, results and discussions (see Sections IV-D, IV-E, VI, Lemma 1, VII and the tabular listing of the proposed JPAUGA algorithm).

The following notations have been used in this paper. $\mathbf{A}^{H}$ and $\mathbf{A}^{T}$ represent conjugate transpose and transpose of the matrix $\mathbf{A}$ respectively. For any complex number $z, z^{*}$ and $|z|$ denote its complex conjugate and absolute value respectively.

\footnotetext{
${ }^{4} \mathrm{~A}$ communication system is said to be in outage if the achieved information sum rate is less than the desired sum rate.
} 


$$
\begin{aligned}
\mathcal{A}_{4}^{2}= & \left\{\left\{\left\{\mathcal{U}_{1}, \mathcal{U}_{2}\right\},\left\{\mathcal{U}_{3}, \mathcal{U}_{4}\right\}\right\},\left\{\left\{\mathcal{U}_{2}, \mathcal{U}_{1}\right\},\left\{\mathcal{U}_{3}, \mathcal{U}_{4}\right\}\right\},\left\{\left\{\mathcal{U}_{1}, \mathcal{U}_{2}\right\},\left\{\mathcal{U}_{4}, \mathcal{U}_{3}\right\}\right\},\left\{\left\{\mathcal{U}_{2}, \mathcal{U}_{1}\right\},\left\{\mathcal{U}_{4}, \mathcal{U}_{3}\right\}\right\},\right. \\
& \left\{\left\{\mathcal{U}_{1}, \mathcal{U}_{3}\right\},\left\{\mathcal{U}_{2}, \mathcal{U}_{4}\right\}\right\},\left\{\left\{\mathcal{U}_{3}, \mathcal{U}_{1}\right\},\left\{\mathcal{U}_{2}, \mathcal{U}_{4}\right\}\right\},\left\{\left\{\mathcal{U}_{1}, \mathcal{U}_{3}\right\},\left\{\mathcal{U}_{4}, \mathcal{U}_{2}\right\}\right\},\left\{\left\{\mathcal{U}_{3}, \mathcal{U}_{1}\right\},\left\{\mathcal{U}_{4}, \mathcal{U}_{2}\right\}\right\}, \\
& \left.\left\{\left\{\mathcal{U}_{1}, \mathcal{U}_{4}\right\},\left\{\mathcal{U}_{3}, \mathcal{U}_{2}\right\}\right\},\left\{\left\{\mathcal{U}_{4}, \mathcal{U}_{1}\right\},\left\{\mathcal{U}_{3}, \mathcal{U}_{2}\right\}\right\},\left\{\left\{\mathcal{U}_{1}, \mathcal{U}_{4}\right\},\left\{\mathcal{U}_{2}, \mathcal{U}_{3}\right\}\right\},\left\{\left\{\mathcal{U}_{4}, \mathcal{U}_{1}\right\},\left\{\mathcal{U}_{2}, \mathcal{U}_{3}\right\}\right\}\right\} .
\end{aligned}
$$

For a random variable $X, \mathbb{E}[X]$ denotes its expected value. The complex and the real fields are denoted by $\mathbb{C}$ and $\mathbb{R}$ respectively. Given a vector $\mathbf{x}=\left(x_{1}, x_{2}, \cdots, x_{n}\right)^{T} \in \mathbb{C}^{n}$, $\|x\| \triangleq \sqrt{\sum_{k=1}^{n}\left|x_{k}\right|^{2}}$. For any two real numbers $x, y \in \mathbb{R}$, $\max (x, y)$ is the maximum between $x$ and $y$. For any real $x$, $[x]^{+} \triangleq \max (x, 0)$. Let $|S|$ denote the cardinality (size) of the set $S$. Given a square matrix $\mathbf{X}$, let $|\mathbf{X}|$ denote its determinant. $\log (x)$ and $\log _{2}(x)$ denote the natural and base-2 logarithm of $x>0$.

\section{SYSTEM MODEL}

Let $\mathbf{H}=\left(\mathbf{h}_{1}, \mathbf{h}_{2}, \cdots, \mathbf{h}_{N_{u}}\right)^{H}$ represent the $N_{u} \times N_{t}$ channel matrix between the base station and the $N_{u}$ single antenna users ${ }^{5}\left(N_{t} \geq N_{u}\right)$. The channel vector from the BS to the $k$-th user is denoted by $\mathbf{h}_{k}^{H} \in \mathbb{C}^{1 \times N_{t}}$, with its $i$-th entry $h_{k, i}^{*}$ representing the channel gain from the $i$-th transmit antenna to the receive antenna of the $k$-th user. ${ }^{6}$ The BS is assumed to have perfect channel state information (CSI). Let $\mathbf{x}=\left(x_{1}, x_{2}, \cdots, x_{N_{t}}\right)^{T} \in \mathbb{C}^{N_{t} \times 1}$ represent the transmitted vector. The vector of received symbols $\mathbf{y}=$ $\left(y_{1}, y_{2}, \cdots, y_{N_{u}}\right)^{T} \in \mathbb{C}^{N_{u} \times 1}$ (with $y_{k}$ denoting the signal received by the $k$-th user) is then given by

$$
\mathbf{y}=\mathbf{H x}+\mathbf{n},
$$

where $\mathbf{n}=\left(n_{1}, n_{2}, \cdots n_{N_{u}}\right)^{T} \in \mathbb{C}^{N_{u} \times 1}$ is the additive noise vector with $n_{k}$ representing the noise at the $k$-th receiver. Further, each entry of $\mathbf{n}$ is an i.i.d. $\mathbb{C N}(0,1)$ random variable. Also, the BS is subject to an average transmit power constraint given by

$$
\mathbb{E}\left[\|\mathbf{x}\|^{2}\right]=P_{T} .
$$

Due to unit variance noise, we will refer to $P_{T}$ as the transmit signal to receiver noise ratio (i.e., transmit SNR). Subsequently we shall refer to the $k$-th user by $\mathcal{U}_{k}$. In the proposed precoding scheme, the total set of users $\mathcal{S}=\left\{\mathcal{U}_{1}, \mathcal{U}_{2}, \cdots \mathcal{U}_{N_{u}}\right\}$ is partitioned into $N_{g}=N_{u} / g$ disjoint groups of size $g$. Let the $i$-th group of users be denoted by the ordered set $\mathcal{S}_{i}=\left\{\mathcal{U}_{i_{1}}, \mathcal{U}_{i_{2}}, \cdots, \mathcal{U}_{i_{g}}\right\}$. Therefore, $\mathcal{S}=\cup_{i=1}^{N_{g}} \mathcal{S}_{i}$, and $\mathcal{S}_{i} \cap \mathcal{S}_{j}=\emptyset, \forall i \neq j$, where $\emptyset$ denotes the null set. Also, let any arbitrary grouping of users be denoted by the unordered set $\mathcal{P}=\left\{\mathcal{S}_{1}, \mathcal{S}_{2}, \cdots, \mathcal{S}_{N_{g}}\right\}$. For example, with $N_{u}=4$ and $g=2$, one possible grouping of users is given by $\mathcal{P}=\left\{\left\{\mathcal{U}_{1}, \mathcal{U}_{4}\right\},\left\{\mathcal{U}_{2}, \mathcal{U}_{3}\right\}\right\}$.

For notational purposes, let us denote the set of all possible groupings of a set of $N_{u}$ users into groups of size $g$, by $\mathcal{A}_{N_{u}}^{g}$.

\footnotetext{
${ }^{5}$ Throughout the paper, $\mathbf{H}$ is assumed to be full rank.

${ }^{6}$ Subsequently we shall also refer to the receiver at the $k$-th user as the $k$-th receiver.
}

For example with $N_{u}=4$ users and $g=2$ we have $\mathcal{A}_{4}^{2}$ as shown in (1). ${ }^{7}$ The number of possible groupings is given by $\left|\mathcal{A}_{N_{u}}^{g}\right|=N_{u} ! /\left(\left(N_{u} / g\right) !\right)$. Let $\mathbf{H}[i] \in \mathbb{C}^{\left(N_{u}-g\right) \times N_{t}}$ denote the sub-matrix of $\mathbf{H}$ consisting of only those rows which represent the channel vector of users not in the set $\mathcal{S}_{i}$, and let $\mathbf{G}[i] \in$ $\mathbb{C}^{g \times N_{t}}$ denote the sub-matrix containing the remaining rows of $\mathbf{H}$. Specifically, if $\mathcal{S}_{i}=\left\{\mathcal{U}_{i_{1}}, \mathcal{U}_{i_{2}}, \cdots, \mathcal{U}_{i_{g}}\right\}$ then

$$
\mathbf{G}[i] \triangleq\left(\mathbf{h}_{i_{1}}, \mathbf{h}_{i_{2}}, \cdots, \mathbf{h}_{i_{g}}\right)^{H} .
$$

Further let $\mathcal{H}_{i}$ represent the subspace spanned by the rows of $\mathbf{H}[i]$, and let $\mathcal{H}_{i}^{\perp}$ be the subspace orthogonal to $\mathcal{H}_{i}$. The projection matrix for the subspace $\mathcal{H}_{i}^{\perp}$ is denoted by

$$
\mathbf{P}[i]=\left(\mathbf{I}_{N_{t}}-\mathbf{H}[i]^{H}\left(\mathbf{H}[i] \mathbf{H}[i]^{H}\right)^{-1} \mathbf{H}[i]\right) \in \mathbb{C}^{N_{t} \times N_{t}} .
$$

Note that $\mathbf{H}[i] \mathbf{P}[i]=0$. Further for the user $\mathcal{U}_{i_{j}}$, let $\mathcal{C}_{i_{j}} \subset \mathbb{C}^{N_{t}}$ denote the space of vectors orthogonal to the space spanned by the rows of $\mathbf{H}[i]$ and the rows of the previous $(j-1)$ users in the $i$-th ordered group $\mathcal{S}_{i}$ (i.e., $\mathbf{h}_{i_{1}}^{H}, \mathbf{h}_{i_{2}}^{H}, \cdots, \mathbf{h}_{i_{j-1}}^{H}$ ).

\section{ZF PRECODER AND THE MOTIVATION FOR GROUPING USERS}

The ZF precoder is a low complexity linear precoder where the information for each user is beamformed in a direction which is orthogonal to the space spanned by the channel vectors of the remaining $N_{u}-1$ users, thereby resulting in no inter-user interference. Hence, for any given user, its effective channel gain is proportional to the Euclidean length of the projection of its channel vector onto the space orthogonal to the space spanned by the channel vectors of remaining users. In case of ill-conditioned channels, since the channel vectors of all the users are "nearly" linearly dependent, the effective channel gain of each user would be small, implying low achievable rates. Therefore it makes sense to design precoders which have a complexity similar to ZF, but which can achieve a higher sum-rate than the ZF precoder when the channel is ill-conditioned.

By grouping users into groups of size larger than one, beamforming can be done to nullify only inter-group interference. Further, it is possible to perform beamforming in such a way that the effective $g \times g$ channel matrix for each group is lower triangular. With small group size and a lower triangular effective channel matrix, intra-group interference can be precancelled using practical successive dirty paper coding (DPC) at the transmitter, without any significant increase in the required transmit power (when compared to an ideal scenario

\footnotetext{
${ }^{7}$ Note that different ordering of users in a group can lead to different information sum rate, and this is why we treat two groups having the same users in a different order, as two different groups.
} 
where the effective channel matrix is diagonal, i.e., no intragroup interference). With this precoding method, the effective channel gain for $\mathcal{U}_{i_{j}}$ would be the Euclidean length of the projection of $\mathbf{h}_{i_{j}}^{H}$ onto the space $\mathcal{C}_{i_{j}}$ (i.e., user $\mathcal{U}_{i_{j}}$ would see interference only from the information symbols of users $\left.\mathcal{U}_{i_{(j+1)}}, \cdots, \mathcal{U}_{i_{g}}\right)$.

On the other hand, with the ZF precoder, the effective channel gain is the Euclidean length of the projection of $\mathbf{h}_{i_{i}}^{H}$ onto the subspace orthogonal to all the rows of $\mathbf{H}$ except $\mathbf{h}_{i_{j}}^{H}$. (We shall subsequently denote this orthogonal subspace by $\mathcal{H}_{i_{j}}^{\perp}$.) It is noted that $\mathcal{H}_{i_{j}}^{\perp} \subset \mathcal{C}_{i_{j}}$ whenever $g>1$. Since the projection of a vector onto a subspace of some space $\mathcal{G}$ is of lesser Euclidean length than its projection onto the space $\mathcal{G}$, it follows that the effective channel gain for $\mathcal{U}_{i_{j}}$ is higher with the proposed user grouping based precoder as compared to that with the ZF precoder. This simple observation coupled with the availability of practical low-complexity DPC for Gaussian broadcast channels with a small number of users, motivates the proposed user grouping based precoder which is presented in Section IV in more detail. For a given user grouping the sum rate is maximized by the waterfilling power allocation across all the users (the effective channel gain of each user is considered).

The sum rate achieved by the proposed precoder is shown to be dependent on the chosen grouping of users. This is expected, as for example with two users having "highly" linearly dependent channel vectors, the information rate to these two users would be higher when they are placed in the same group. Therefore in Section V we propose to jointly maximize the sum rate of the proposed precoder w.r.t. the power allocation and the possible user groupings.

\section{Proposed User Grouping based Precoder}

This section is organized into several subsections. For a given user grouping $\mathcal{P}$, we beamform information symbols in such a way that only inter-group interference is nullified. With the proposed beamforming the original $N_{u}$-user Gaussian broadcast channel is transformed into $N_{g}$ parallel $g$-user Gaussian broadcast channels. This is presented in Section IV-A, where we finally show that the proposed multiuser beamforming is such that the effective channel matrix for each group is lower triangular. Subsequently in Section IV-B, using the fact that the effective channel is lower triangular we use Dirty Paper Coding to cancel interference between the users within a group. We also show that for a fixed user grouping, the information sum rate is maximized by the waterfilling power allocation. In Section IV-C we show that the ZF precoder is a special case of the proposed precoder with $N_{u}$ groups, i.e., $g=1$. We also present expressions for the sum rate achieved by the ZF precoder. Next, in Section IV-D we analytically show that the proposed precoder with any arbitrary grouping having $g \geq 2$ always achieves a higher information sum rate than the ZF precoder irrespective of the channel realization $\mathbf{H}$ and $P_{T}$. Finally, in Section IV-E we present an example to demonstrate the higher sum rate achieved by the proposed precoder in comparison with the ZF precoder, with random user grouping (i.e., the user grouping is chosen independent of the CSI). Through another example we show that random user grouping is sub-optimal, and this motivates the problem of finding the optimal user grouping which is discussed in Section V.

\section{A. Beamforming to cancel inter-group interference}

Let $\mathbf{u}[i] \triangleq\left(u_{i_{1}}, u_{i_{2}}, \cdots, u_{i_{g}}\right)^{T}$ be the $g \times 1$ vector of information symbols of the users in the $i$-th group $\mathcal{S}_{i}$. The information symbols are assumed to be i.i.d. Gaussian distributed with mean 0 and variance 1 . The proposed precoder maps $\mathbf{u}[i]$ onto $\mathbf{x}[i] \in \mathbb{C}^{N_{t} \times 1}$ through the linear transformation

$$
\mathbf{x}[i]=\mathbf{D}[i] \mathbf{u}[i],
$$

where $\mathbf{D}[i] \in \mathbb{C}^{N_{t} \times g}$ is the precoding matrix for the $i$-th group of users. The vector transmitted from the BS is then given by

$$
\mathbf{x}=\sum_{i=1}^{N_{g}} \mathbf{x}[i] .
$$

Note that the transmit power constraint in (3) requires that the precoding matrices satisfy the constraint

$$
\sum_{i=1}^{N_{g}}\|\mathbf{D}[i]\|_{F}^{2}=P_{T}
$$

where $\|\mathbf{X}\|_{F}$ denotes the Frobenius norm of the matrix $\mathbf{X}$.

Let $\mathbf{y}[i] \triangleq\left(y_{i_{1}}, y_{i_{2}}, \cdots, y_{i_{g}}\right)^{T}$ be the $g \times 1$ vector of symbols received by the users in the $i$-th group $\mathcal{S}_{i}$. Using (2), (6) and (7), the received vector $\mathbf{y}[i]$ is given by

$$
\begin{aligned}
\mathbf{y}[i] & =\mathbf{G}[i]\left(\mathbf{x}[i]+\sum_{k=1, k \neq i}^{N_{g}} \mathbf{x}[k]\right)+\mathbf{n}[i] \\
& =\mathbf{G}[i] \mathbf{D}[i] \mathbf{u}[i]+\sum_{k=1, k \neq i}^{N_{g}} \mathbf{G}[i] \mathbf{D}[k] \mathbf{u}[k]+\mathbf{n}[i] .
\end{aligned}
$$

In (9), the term $\sum_{k=1, k \neq i}^{N_{g}} \mathbf{G}[i] \mathbf{D}[k] \mathbf{u}[k]$ corresponds to the interference to the users in the $i$-th group due to signals transmitted by the BS for the other $\left(N_{g}-1\right)$ remaining groups. This interference can be nullified by choosing the precoding matrix $\mathbf{D}[k]$ for the $k$-th group in such a way that its columns are orthogonal to the channel vectors of all the users in the other groups. One way of achieving this as well as the power constraint in (8) is to have

$$
\mathbf{D}[k]=\mathbf{Q}[k] \mathbf{W}[k], k=1, \ldots, N_{g},
$$

where $\mathbf{Q}[k] \in \mathbb{C}^{N_{t} \times g}$ is the matrix whose columns form an orthonormal basis for the subspace $\mathcal{H}_{k}^{\perp}$ (i.e., the subspace of vectors orthogonal to the channel vectors of all users in the other groups except $\mathcal{S}_{k}$ ). The matrix $\mathbf{W}[k]=$ $\operatorname{diag}\left(\sqrt{p_{k_{1}}}, \sqrt{p_{k_{2}}}, \cdots \sqrt{p_{k_{g}}}\right)$, is the diagonal power allocation matrix for the users in the $k$-th group with $p_{k_{j}}$ being the power allocated to the information symbol of $\mathcal{U}_{k_{j}}$. Therefore by design, we have $\mathbf{G}[i] \mathbf{Q}[k]=0$ for all $i \neq k$, since for any $i \neq k$ the rows of $\mathbf{G}[i]$ (i.e., channel vectors of users in the $i$-th group) belong to the subspace $\mathcal{H}_{k}$ and the columns of 
$\mathbf{Q}[k]$ are orthogonal to any vector in $\mathcal{H}_{k}$. This then implies that $\mathbf{G}[i] \mathbf{D}[k]=0$ for all $i \neq k$. Using this fact in (9) we get

$$
\mathbf{y}[i]=\mathbf{B}[i] \mathbf{u}[i]+\mathbf{n}[i]
$$

where

$$
\mathbf{B}[i] \triangleq \mathbf{G}[i] \mathbf{Q}[i] \mathbf{W}[i]
$$

is the $g \times g$ effective channel gain matrix for the $i$-th group of users. From (11) it is clear that each group of users does not have any interference from the other groups. Essentially the original $N_{u}$ user MISO broadcast channel has been decomposed into $N_{g}$ parallel non-interfering $g$-user MISO broadcast subchannels.

For the $i$-th group of users an orthonormal basis for the subspace $\mathcal{H}_{i}^{\perp}$ (i.e., columns of $\mathbf{Q}[i]$ ) can be found through the QR decomposition [26] of the matrix $\mathbf{F}[i] \triangleq \mathbf{P}[i] \mathbf{G}[i]^{H}$ which is given by

$$
\mathbf{F}[i]=\mathbf{Q}[i] \mathbf{R}[i]
$$

Here $\mathbf{R}[i] \in \mathbb{C}^{g \times g}$ is an upper triangular matrix with positive diagonal entries (since $\mathbf{F}[i]$ is full rank), and $\mathbf{Q}[i] \in \mathbb{C}^{N_{t} \times g}$ is a matrix with orthonormal columns. The $g$ orthonormal columns of $\mathbf{Q}[i]$ form an orthonormal basis for the space $\mathcal{H}_{i}^{\perp}$ since $\mathbf{H}[i] \mathbf{Q}[i] \mathbf{R}[i]=\mathbf{H}[i] \mathbf{F}[i]=\mathbf{H}[i] \mathbf{P}[i] \mathbf{G}[i]^{H}=0$ and therefore $\mathbf{H}[i] \mathbf{Q}[i]=0$.

Using (10) along with the fact that the columns of $\mathbf{Q}[k]$ are orthonormal, the sum power constraint in (8) is given by

$$
\begin{aligned}
\sum_{i=1}^{N_{g}}\left\|\mathbf{D}_{i}\right\|_{F}^{2} & =\sum_{i=1}^{N_{g}}\left\|\mathbf{Q}_{i} \mathbf{W}_{i}\right\|_{F}^{2} \\
& =\sum_{i=1}^{N_{g}} \operatorname{Tr}\left(\mathbf{W}_{i}^{H} \mathbf{Q}_{i}^{H} \mathbf{Q}_{i} \mathbf{W}_{i}\right) \\
& =\sum_{i=1}^{N_{g}} \operatorname{Tr}\left(\mathbf{W}_{i}^{H} \mathbf{W}_{i}\right)=\sum_{i=1}^{N_{g}} \sum_{j=1}^{g} p_{i_{j}}=P_{T},
\end{aligned}
$$

where we have used the fact that $\mathbf{Q}[i]$ has orthonormal columns and $\operatorname{Tr}(\cdot)$ denotes the trace operation for matrices. Subsequently, let $\mathbf{p}=\left(p_{1}, p_{2}, \cdots, p_{N_{u}}\right)$ denote the power allocation vector, with $p_{i}$ being the power allocated to $\mathcal{U}_{i}$. We next show that the effective channel gain matrix $\mathbf{B}[i]$ is a lower triangular matrix and is equal to $\mathbf{R}[i]^{H} \mathbf{W}[i]$. From the definitions of $\mathbf{P}[i]$ and $\mathbf{Q}[i]$ in (5) and (13), it is clear that $\mathbf{P}[i]$ is the projection matrix for $\mathcal{H}_{i}^{\perp}$ which is also the space spanned by the columns of $\mathbf{Q}[i]$ and therefore

$$
\mathbf{P}[i] \mathbf{Q}[i]=\mathbf{Q}[i] .
$$

Since $\mathbf{F}[i]=\mathbf{Q}[i] \mathbf{R}[i]=\mathbf{P}[i] \mathbf{G}[i]^{H}$, we have

$$
\begin{aligned}
\mathbf{R}[i] & =\mathbf{Q}[i]^{H}(\mathbf{Q}[i] \mathbf{R}[i]) \stackrel{(\text { a) }}{=} \mathbf{Q}[i]^{H} \mathbf{F}[i] \\
& =\mathbf{Q}[i]^{H} \mathbf{P}[i] \mathbf{G}[i]^{H} \stackrel{(\mathrm{b})}{=} \mathbf{Q}[i]^{H} \mathbf{P}[i]^{H} \mathbf{G}[i]^{H} \\
& =(\mathbf{P}[i] \mathbf{Q}[i])^{H} \mathbf{G}[i]^{H} \stackrel{(\mathrm{c})}{=} \mathbf{Q}[i]^{H} \mathbf{G}[i]^{H},
\end{aligned}
$$

where step (a) follows from (13), step (b) follows from the fact that $\mathbf{P}[i]$ is Hermitian and step (c) follows from (15). Using (16) in (12) we see that $\mathbf{B}[i]=\mathbf{R}[i]^{H} \mathbf{W}[i]$, i.e., the effective channel is lower triangular. Using this expression for $\mathbf{B}[i]$ in (11) we have

$$
\mathbf{y}[i]=\mathbf{R}[i]^{H} \mathbf{W}[i] \mathbf{u}[i]+\mathbf{n}[i]
$$

From (17), the received signal at the $j$-th user in the $i$-th group is given by

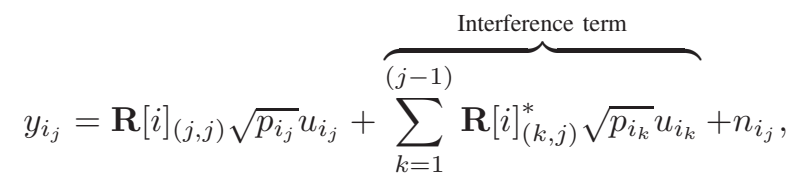

where $j=1,2, \ldots, g$ and $\mathbf{R}[i]_{(k, j)}$ denotes the entry of $\mathbf{R}[i]$ in the $k$-th row and the $j$-th column. Due to the lower triangular structure of the effective channel matrix for the $i$-th group, from (18), we observe that the $j$-th user in the $i$-th group (i.e., $\mathcal{U}_{i_{j}}$ ) has interference only from the symbols of the previous $(j-1)$ users in the same group (i.e., $\left.\mathcal{U}_{i_{1}}, \cdots \mathcal{U}_{i_{(j-1)}}\right)$.

\section{B. Dirty Paper Coding to cancel intra-group interference}

In the proposed coding scheme, for the $i$-th group, we start with precoding information for the first user $\mathcal{U}_{i_{1}}$, and since it sees no interference from any other user, we simply use an AWGN channel code with rate

$$
r_{i_{1}}=\log _{2}\left(1+p_{i_{1}} \mathbf{R}[i]_{(1,1)}^{2}\right) .
$$

From (18) it is clear that the second user $\mathcal{U}_{i_{2}}$, has an interference term with contribution only from the first user $\mathcal{U}_{i_{1}}$. Since the BS has perfect CSI and it knows the transmitted information symbol for the first user (i.e., $u_{i_{1}}$ ), it knows the interference term for the second user, and can therefore perform known interference pre-cancellation using the Dirty Paper Coding scheme [19], [20], [21]. In a similar manner, for the $j$-th user $\mathcal{U}_{i_{j}}$, the BS can perform Dirty Paper Coding for the known interference term which has contributions only from the previously precoded $(j-1)$ users $\left(\mathcal{U}_{i_{1}}, \mathcal{U}_{i_{2}}, \cdots, \mathcal{U}_{i_{(j-1)}}\right)$. The rate achieved by the $j$-th user in the $i$-th group is therefore given by

$$
r_{i_{j}}=\log _{2}\left(1+p_{i_{j}} \mathbf{R}[i]_{(j, j)}^{2}\right), j=2,3, \ldots, g .
$$

For a given grouping of users $\mathcal{P} \in \mathcal{A}_{N_{u}}^{g}$, total power constraint $P_{T}$, channel realization $\mathbf{H}$ and power allocation vector $\mathbf{p}$, the sum rate achieved by the proposed precoder is therefore given by

$$
\begin{aligned}
r\left(\mathbf{H}, P_{T}, \mathcal{P}, \mathbf{p}\right) & \triangleq \sum_{k=1}^{N_{u} / g} \sum_{j=1}^{g} r_{k_{j}} \\
& =\sum_{k=1}^{N_{u} / g} \sum_{j=1}^{g} \log _{2}\left(1+p_{k_{j}} \mathbf{R}[k]_{(j, j)}^{2}\right) .
\end{aligned}
$$

Maximization of $r\left(\mathbf{H}, P_{T}, \mathcal{P}, \mathbf{p}\right)$ over $\mathbf{p}$ yields

$$
r\left(\mathbf{H}, P_{T}, \mathcal{P}\right) \triangleq \max _{\mathbf{p} \mid \sum_{i=1}^{N_{u}} p_{i}=P_{T}, p_{i} \geq 0} r\left(\mathbf{H}, P_{T}, \mathcal{P}, \mathbf{p}\right)
$$


In (22), the optimal power allocation for a given grouping of users is given by the waterfilling scheme [22], i.e.

$$
p_{k_{j}}=\left[\mu-\frac{1}{\mathbf{R}[k]_{(j, j)}^{2}}\right]^{+},
$$

where $k=1,2, \ldots, N_{u} / g, j=1,2, \ldots, g$ and $\mu>0$ is such that

$$
\sum_{k=1}^{N_{u} / g} \sum_{j=1}^{g} p_{k_{j}}=P_{T}
$$

C. The ZF precoder: A special case of the proposed precoder

We note that the ZF precoder is a special case of the proposed user grouping scheme with $g=1$, i.e., $N_{u}$ groups with one user per group. Subsequently, for $g=1$ (i.e, the ZF precoder), we shall denote the optimal waterfilling power allocation (given by (23) and (24)) by $\mathbf{p}^{*}=\left(p_{1}^{*}, p_{2}^{*}, \cdots, p_{N_{u}}^{*}\right)$. The sum rate achieved by the ZF precoder can be shown to be

$$
C_{\mathrm{ZF}}\left(\mathbf{H}, P_{T}\right)=\sum_{i=1}^{N_{u}} \log _{2}\left(1+\frac{p_{i}^{*}}{\left[\left(\mathbf{H H}^{H}\right)^{-1}\right]_{(i, i)}}\right),
$$

where $\mathbf{p}^{*}$ is given by

$$
p_{i}^{*}=\left[\lambda-\left[\left(\mathbf{H H}^{H}\right)^{-1}\right]_{(i, i)}\right]^{+}, \forall i=1,2, \ldots, N_{u} .
$$

The variable $\lambda>0$ is chosen such that

$$
\sum_{i=1}^{N_{u}} p_{i}^{*}=P_{T}
$$

The other special case is for $g=N_{u}$, i.e., only one group consisting of all the $N_{u}$ users. This has been discussed in detail in [20] as the ZF-DP precoder.

\section{$D$. The proposed precoder achieves a higher information rate than the $Z F$ precoder}

The following theorem shows that irrespective of the channel realization $\mathbf{H}$ and $P_{T}$, the sum rate achieved by the proposed precoder with any arbitrary user grouping having $g \geq 2$ is greater than that achieved by the $\mathrm{ZF}$ precoder (i.e., proposed precoder with $g=1$ ).

Theorem 4.1: Let $\mathcal{P} \in \mathcal{A}_{N_{u}}^{g}$ be any arbitrary user grouping with $g \geq 2$. Then

$$
r\left(\mathbf{H}, P_{T}, \mathcal{P}\right) \geq C_{\mathrm{ZF}}\left(\mathbf{H}, P_{T}\right)
$$

holds for any channel realization $\mathbf{H}$ and $P_{T}$.

Proof - See Appendix A.

In the following we illustrate the effectiveness of the proposed idea of grouping users through an example where for a Rayleigh fading channel we show that for any $P_{T}$ the ergodic sum rate (i.e, sum rate averaged over all realizations of $\mathbf{H}$ ) achieved by the proposed precoder (with $g=2$ and random user grouping) is always greater than that achieved by the $\mathrm{ZF}$ precoder. We will also show that to achieve a given fixed sum rate, the ZF precoder asymptotically (i.e., as $P_{T} \rightarrow \infty$ ) requires about $2.17 \mathrm{~dB}$ more power than the proposed precoder (with $g=2$ and random user grouping).
Example 1: Let $N_{t}=N_{u}$ and the entries of $\mathbf{H}$ be i.i.d. Rayleigh faded with each entry distributed as a circular symmetric complex Gaussian random variable having zero mean and unit variance. Let

$$
d\left(P_{T}, N_{u}\right) \triangleq \mathbb{E}_{\mathbf{H}}\left[r\left(\mathbf{H}, P_{T}, \mathcal{P}, \mathbf{p}\right)-C_{\mathrm{ZF}}\left(\mathbf{H}, P_{T}\right)\right]
$$

denote the difference between the ergodic sum rates achieved by the ZF precoder and that achieved by the proposed precoder (with $g=2$ ). Further, for the proposed precoder, let the user pairs (since $g=2$ ) be formed randomly (random grouping), i.e., the pairing of users is assumed to be independent of the channel realization $\mathbf{H}$. The power allocation vector $\mathbf{p}$ for the proposed precoder is assumed to be uniform, i.e., $p_{i}=P_{T} / N_{u}, i=1,2, \ldots, N_{u}{ }^{8}{ }^{8}$

Lemma 1: Under the above assumptions, $d\left(P_{T}, N_{u}\right)$ can be bounded as follows

$$
\begin{aligned}
\frac{N_{u}}{2} \log _{2}(e)(1 & \left.-\frac{N_{u}}{P_{T}} \log \left(1+\frac{P_{T}}{N_{u}}\right)\right)<d\left(P_{T}, N_{u}\right) \\
& <\frac{N_{u}}{2} \log _{2}(e)\left(1-\frac{N_{u}}{2 P_{T}} \log \left(1+\frac{2 P_{T}}{N_{u}}\right)\right)
\end{aligned}
$$

Proof - See Appendix B.

Remark 1: We firstly note that both the upper and lower bounds in (30) are strictly positive for all $P_{T}>0$. This is because $g(x) \triangleq x-\log (1+x)$ is strictly positive for all $x>0$, and the lower and upper bounds in (30) are $\frac{g\left(P_{T} / N_{u}\right)}{P_{T} / N_{u}}$ and $\frac{g\left(2 P_{T} / N_{u}\right)}{2 P_{T} / N_{u}}$ respectively. ${ }^{9}$ For a fixed $N_{t}=N_{u}$, the lower and upper bounds in (30) can be shown to converge to $N_{u} \log _{2}(e) / 2$ as $P_{T} \rightarrow \infty$, which implies that at sufficiently high SNR, by randomly pairing users the proposed precoder can achieve an ergodic sum rate which is $N_{u} \log _{2}(e) / 2$ bits per channel use (bpcu) greater than the ergodic sum rate achieved by the ZF precoder. Further, at high SNR the slope of the sum rate achieved by the $\mathrm{ZF}$ precoder w.r.t. $\log \left(P_{T}\right)$ is $N_{u} \log _{2}(e)$. This then implies that at high SNR, the ZF precoder needs roughly $10 \log _{10}(\sqrt{e})=2.17 \mathrm{~dB}$ more power than that required by the proposed precoder with $(g=2$, random grouping) to achieve a given ergodic sum rate. An important observation on this result is that, the asymptotic SNR gap of $2.17 \mathrm{~dB}$ is independent of $N_{u}$.

The above analysis shows that, even with random user grouping, the proposed grouping based precoder is more power efficient than the ZF precoder.

\section{E. Motivating the need for "optimal" user grouping}

So far we have not bothered much about the choice of user grouping. The following example shows the sensitivity of the proposed precoder w.r.t. the chosen user grouping. This then

\footnotetext{
${ }^{8}$ It is to be noted that this is justified at high $\operatorname{SNR}\left(P_{T} \rightarrow \infty\right)$ since the optimal waterfilling power allocation is almost the same as uniform power allocation

${ }^{9}$ Note that $g(x=0)=0$ and its first derivative $\frac{d g(x)}{d x}=\frac{x}{1+x}>0$ for all $x>0$. This implies that $g(x)>0$ for all $x>0$.
} 


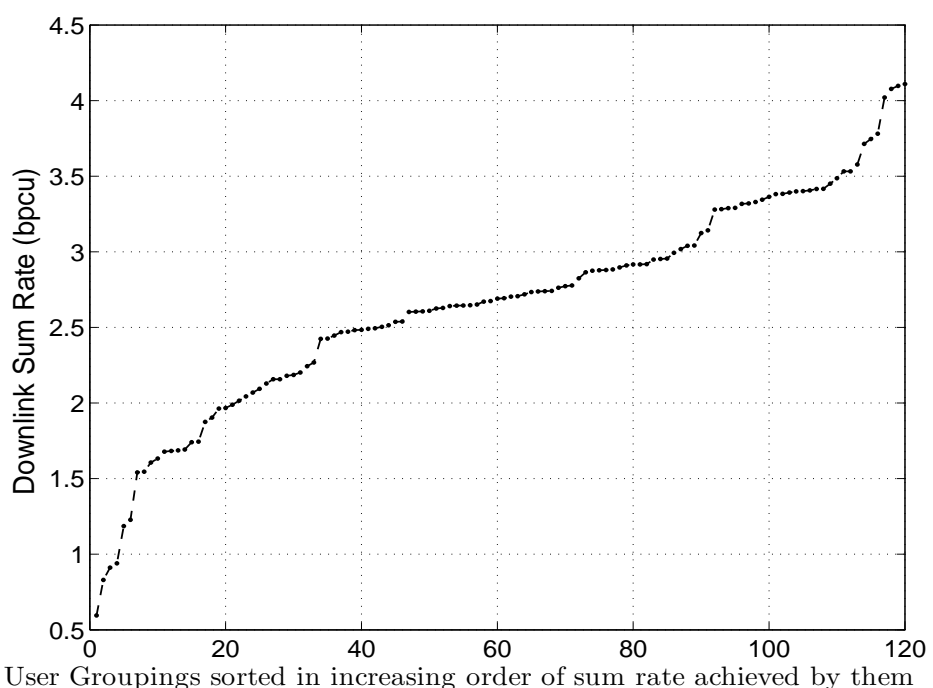

Fig. 1. Sensitivity of the achievable sum rate towards the chosen grouping of users for the MISO broadcast channel given by (31). Here $N_{t}=N_{u}=6$, $g=2$ and $P_{T}=10 \mathrm{~dB}$. The number of possible groupings is $\left|\mathcal{A}_{6}^{2}\right|=120$.

motivates us to choose the user grouping which maximizes the sum rate.

Example 2: In this example we consider a $N_{t}=N_{u}=$ 6 Gaussian broadcast channel whose channel matrix is illconditioned and is given by

$$
\mathbf{H}_{e x}=\left[\begin{array}{cccccc}
\frac{1}{2} & 0 & 0 & -\frac{1}{2} & \frac{1}{\sqrt{2}} & 0 \\
0 & \frac{1}{2} & -\frac{1}{\sqrt{2}} & \frac{1}{2} & 0 & 0 \\
0 & -\frac{1}{2} & 0 & 0 & -\frac{1}{\sqrt{2}} & \frac{1}{2} \\
-\frac{1}{2} & 0 & 0 & \frac{1}{\sqrt{2}} & 0 & -\frac{1}{2} \\
\frac{1}{2} & 0 & \frac{1}{\sqrt{2}} & 0 & \frac{1}{2} & 0 \\
0 & 0 & 0 & -\frac{1}{\sqrt{2}} & -\frac{1}{2} & \frac{1}{2}
\end{array}\right] \text {. }
$$

The ordered singular values of $\mathbf{H}_{e x}$ are $(1.56,1.48,0.97,0.54,0.38,0.028)$. For the channel in (31) we compute the sum rate $r\left(\mathbf{H}, P_{T}, \mathcal{P}\right)$ achieved by the proposed precoder with each of the $\left|\mathcal{A}_{6}^{2}\right|=120$ different user groupings $\mathcal{P} \in \mathcal{A}_{6}^{2}$ ( $P_{T}$ is fixed to $10 \mathrm{~dB}$ ). For a given grouping of users, power allocation is given by the optimal waterfilling scheme in (23) and (24). We then order the user groupings in increasing order of the sum rates achieved by them, i.e., the ordered user grouping number 1 achieves the least sum rate and the ordered user grouping number 120 achieves the largest sum rate. In Fig. 1, we plot the sum rate (vertical axis) versus the ordered user grouping number (horizontal axis). From the figure, it is observed that there is a wide variation in the achievable information rate. There are about 20 out of 120 user groupings for which the sum rate is below 2 bpcu. At the same time more than 10 user groupings achieve a sum rate greater than 3.5 bpcu. This shows the sensitivity of the achievable sum rate towards the chosen grouping of users.

Motivated by the sensitivity of the proposed precoder w.r.t. user grouping we define the optimal user grouping as one which maximizes the sum rate. The optimal user grouping is

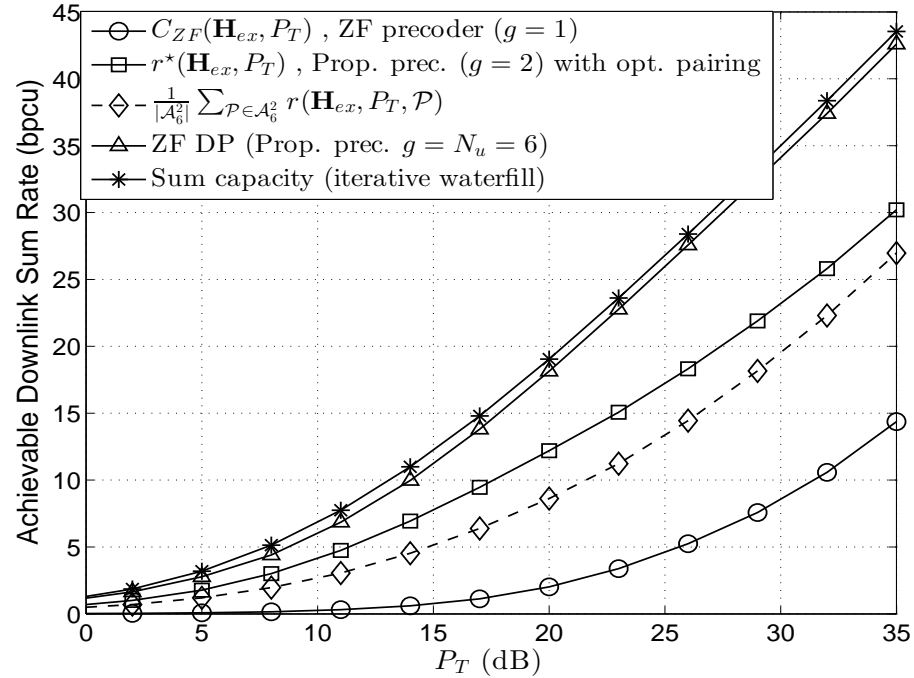

Fig. 2. Comparison of the sum rates achieved by the proposed user grouping precoder and the ZF precoder for the broadcast channel in (31).

clearly a function of $\left(\mathbf{H}, P_{T}\right)$ and is given by

$$
\mathcal{P}^{\star}\left(\mathbf{H}, P_{T}\right) \triangleq \arg \max _{\mathcal{P} \in \mathcal{A}_{N_{u}}^{g}} r\left(\mathbf{H}, P_{T}, \mathcal{P}\right),
$$

where $r\left(\mathbf{H}, P_{T}, \mathcal{P}\right)$ is given by (22). From (32) it follows that for a given $\left(\mathbf{H}, P_{T}\right)$ the maximum information sum rate is given by the value of $r\left(\mathbf{H}, P_{T}, \mathcal{P}\right)$ evaluated for the optimal user grouping $\mathcal{P}=\mathcal{P}^{\star}\left(\mathbf{H}, P_{T}\right)$. This maximum information sum rate is denoted by

$$
r^{\star}\left(\mathbf{H}, P_{T}\right) \triangleq r\left(\mathbf{H}, P_{T}, \mathcal{P}^{\star}\left(\mathbf{H}, P_{T}\right)\right) .
$$

For the $6 \times 6$ channel in (31), we numerically compute the optimal user grouping for the proposed precoder with $g=$ 2 and compare the resulting optimal sum rate with the sum rate achieved by the $\mathrm{ZF}$ precoder i.e., $C_{\mathrm{ZF}}\left(\mathbf{H}_{e x}, P_{T}\right)$. This comparison is depicted graphically as a function of $P_{T}$ in Fig. 2. We also plot the information sum rate of the proposed precoder averaged over all possible groupings (see the curve marked with diamonds). ${ }^{10}$ It is observed that indeed optimal user grouping results in significant improvement in sum rate. As an example, at $P_{T}=10 \mathrm{~dB}$ the information sum rate of the ZF precoder is only $0.31 \mathrm{bpcu}$ when compared to 4.75 bpcu achieved by the proposed precoder with optimal user grouping. Also with random user grouping (curve marked with diamonds) the average information sum rate achieved by the proposed precoder is $3 \mathrm{bpcu}$ at $P_{T}=10 \mathrm{~dB}$. Therefore, in ill-conditioned channels it appears that choosing the optimal grouping can lead to significant improvement in the sum rate performance of the proposed precoder. Note that the sum rate of the proposed user grouping scheme is significantly higher than that of the ZF precoder even for small $g=2$. Exhaustive simulations have revealed that the sum rate of the proposed user grouping scheme increases with increasing $g$.

\footnotetext{
${ }^{10}$ In Fig. 2, $r\left(\mathbf{H}_{e x}, P_{T}, \mathcal{P}\right)$ is $r\left(\mathbf{H}, P_{T}, \mathcal{P}\right)$ (see (22)) evaluated for the ill-conditioned channel $\mathbf{H}=\mathbf{H}_{e x}$ given by (31). The curve marked with dashed diamonds is the average value of the information rates achieved by all the $\left|\mathcal{A}_{6}^{2}\right|=120$ possible user groupings/pairings (since $g=2$ ).
} 
In Fig. 2 we also plot the sum capacity ${ }^{11}$ of the multiuser channel in (31) and the sum rate achieved by the ZF-DP precoding scheme (i.e., special case of the proposed user grouping scheme with $g=N_{u}=6$ ). We observe that the ZF-DP scheme is near sum capacity achieving and has a better sum rate performance than the proposed user grouping precoder with $g=2$ (optimal pairing). However, the ZFDP precoder achieves this better performance at the cost of a significantly higher complexity when compared to the proposed user grouping precoder with $g=2$, as is discussed in the following.

In ZF-DP (i.e., proposed user grouping precoder with $g=$ $\left.N_{u}\right)$ successive DPC has to be performed for $\left(N_{u}-1\right)$ users, whereas when $g=2$ successive DPC needs to be performed for only $N_{u} / 2$ users (only for the second user in each group). With successive DPC, the power of the known interference signal due to other users will increase with the user index, i.e., the first user to be precoded will not see any interference, the second user will see interference only from the first user, the third user will see interference from both the first and the second user, and so on [20]. With $g=2$, DPC is performed only for the second user in each group, and therefore the interference power is roughly of the same order as the power of the useful information symbol. On the other hand for ZF-DP $\left(g=N_{u}\right)$, the last user to be precoded needs to perform DPC for interference from all the previous $\left(N_{u}-1\right)$ users. Hence the interference power for each successive DPC is expected to be higher for the ZF-DP precoder in comparison to the proposed precoder with $g=2$. This larger interference power will lead to increase in complexity of known practical nearoptimal-DPC schemes. As an example, in [8] it is mentioned that with increasing interference power the size of the channel code alphabet set (constellation) has to be increased in order to ensure that the interference signal lies entirely inside the expanded constellation. This expansion in the constellation will also increase the dynamic range of the received signal at the user end, which can then increase the design complexity of the receiver. In general it is expected that increasing $g$ will increase the sum rate performance of the proposed precoder, but at the cost of higher complexity. ${ }^{12}$

\section{PARTITIONING USERS INTO GROUPS}

For small $N_{u}$, (32) can be solved simply by brute-force enumeration of all possible groupings. However, for large $N_{u}$, the combinatorial nature of the problem makes it inherently complex to solve by brute-force enumeration. ${ }^{13}$ Therefore for large $N_{u}$ we propose an iterative "Joint Power Allocation and User Grouping Algorithm" (JPAUGA), which solves (32)

\footnotetext{
${ }^{11}$ The sum capacity of the broadcast channel is computed using the sum power iterative waterfilling method proposed in [4].

${ }^{12} \mathrm{An}$ exact quantitative complexity comparison between ZF-DP and the proposed user grouping precoder with $g=2$ is difficult. This is because, the complexity of performing DPC is different for either case due to the difference in the interference power level for each successive DPC for which different constellations must be used. Further, the complexity of performing practical DPC is also implementation dependent (e.g., LDPC, convolutional codes).

${ }^{13}$ The number of possible groupings, i.e., $\left|\mathcal{A}_{N_{u}}^{g}\right|=N_{u} ! /\left(\left(N_{u} / g\right) !\right)$ grows exponentially with $N_{u}$ for a fixed $g$. For example with $g=2$ and even $N_{u},\left|\mathcal{A}_{N_{u}}^{g}\right|=2^{N_{u} / 2}\left(N_{u}-1\right) \cdot\left(N_{u}-3\right) \cdots 3 \cdot 1$.
}

approximately. Numerical results demonstrate that JPAUGA achieves an information rate close to the optimal $r^{\star}\left(\mathbf{H}, P_{T}\right)$.

Let $\mathcal{P}^{(q)}$ be the user grouping after the $q$-th iteration of JPAUGA. Similarly, let $\mathbf{p}^{(q)}$ be the power allocation after the $q$-th iteration of JPAUGA. JPAUGA starts with initializing the power allocation to be the ZF power allocation i.e., $\mathbf{p}^{(0)}=\mathbf{p}^{*}$ (see Section IV-C). In the $q$-th iteration $\left(q=1,2, \ldots, \max _{i t r}\right)$, we firstly find the user grouping $\mathcal{P}^{(q)}$ which approximately maximizes the information sum rate with power allocation fixed to its values at the end of the $(q-1)$-th iteration, i.e., $\mathbf{p}=\mathbf{p}^{(q-1)}$. That is, $\mathcal{P}^{(q)}$ is an approximate solution to the problem

$$
\arg \max _{\mathcal{P} \in \mathcal{A}_{N_{u}}^{g}} r\left(\mathbf{H}, P_{T}, \mathcal{P}, \mathbf{p}^{(q-1)}\right) .
$$

In Section V-A we propose an approximate solution to (34), called "Generalized User Grouping Algorithm" (GUGA). After computing $\mathcal{P}^{(q)}$ using GUGA, the power allocation for the $q$-th iteration, i.e., $\mathbf{p}^{(q)}$ is given by the waterfilling scheme with user grouping fixed to $\mathcal{P}^{(q)}$ (see (23) and (24)). The proposed iterative algorithm JPAUGA then moves to the $(q+1)$-th iteration.

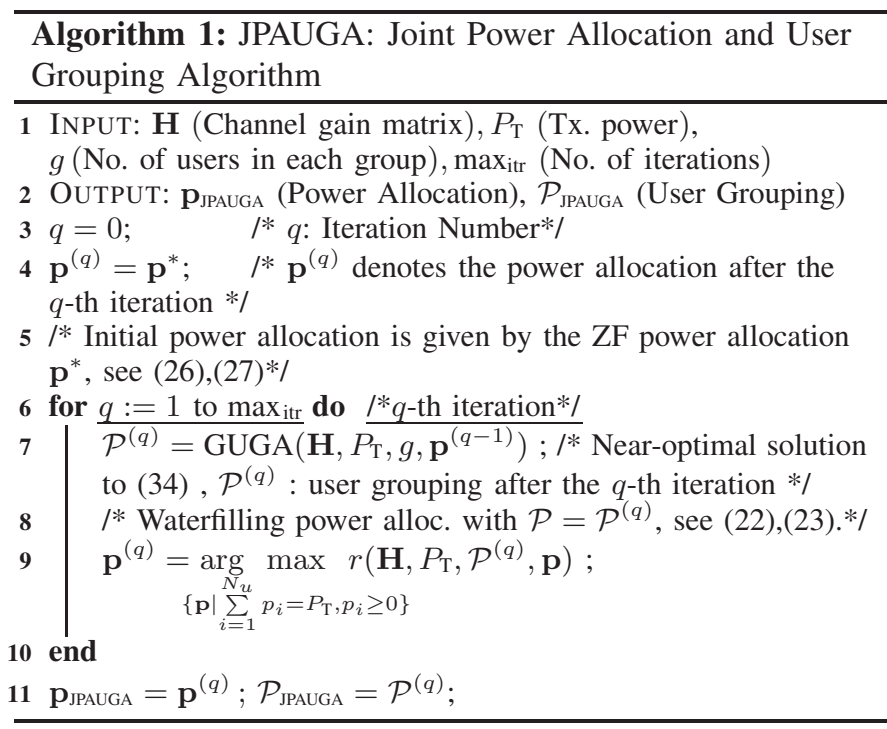

Due to alternating maximization of the information sum rate w.r.t. user grouping and power allocation, it is clear that the information sum rate increases successively from one iteration to the next, i.e., $r\left(\mathbf{H}, P_{T}, \mathcal{P}^{(q+1)}, \mathbf{p}^{(q+1)}\right) \geq$ $r\left(\mathbf{H}, P_{T}, \mathcal{P}^{(q)}, \mathbf{p}^{(q)}\right)$. The algorithm terminates either after a fixed number of iterations (e.g., $\max _{i t r}$ ) or till the relative iteration-by-iteration improvement in the information sum rate i.e., $\left[r\left(\mathbf{H}, P_{T}, \mathcal{P}^{(q+1)}, \mathbf{p}^{(q+1)}\right)-\right.$ $\left.r\left(\mathbf{H}, P_{T}, \mathcal{P}^{(q)}, \mathbf{p}^{(q)}\right)\right] / r\left(\mathbf{H}, P_{T}, \mathcal{P}^{(q)}, \mathbf{p}^{(q)}\right)$ falls below a certain pre-determined threshold. The JPAUGA algorithm is summarized in the table above. The GUGA algorithm (see line 7 in the algorithm above) is discussed in the following section. 


\section{A. Generalized User Grouping Algorithm - GUGA}

In this section we discuss the problem of finding the user grouping which maximizes the information sum rate for a fixed $\left(\mathbf{H}, P_{T}, \mathbf{p}\right)$, i.e.,

$$
\arg \max _{\mathcal{P} \in \mathcal{A}_{N_{u}}^{g}} r\left(\mathbf{H}, P_{T}, \mathcal{P}, \mathbf{p}\right) .
$$

This problem is combinatorial in nature and it appears that finding the optimal user grouping would be prohibitive for large $N_{u}$. Therefore in the following we propose a low complexity approximate solution to (35), called "GUGA".

Before discussing GUGA in detail, for any arbitrary user grouping $\mathcal{P}=\left\{\mathcal{S}_{1}, \cdots, \mathcal{S}_{N_{g}}\right\}$ we define the rate of the $k$-th group of $g$ users i.e., $\mathcal{S}_{k}=\left\{\mathcal{U}_{k_{1}}, \mathcal{U}_{k_{2}}, \cdots, \mathcal{U}_{k_{g}}\right\}$ by ${ }^{14}$

$$
\mathcal{I}\left(\mathcal{S}_{k}\right) \triangleq \sum_{j=1}^{g} \log _{2}\left(1+p_{k_{j}} \mathbf{R}[k]_{(j, j)}^{2}\right) .
$$

The optimization problem in (35) can therefore be expressed as

$$
\arg \max _{\mathcal{P}=\left\{\mathcal{S}_{1}, \mathcal{S}_{2}, \cdots, \mathcal{S}_{N_{g}}\right\} \in \mathcal{A}_{N_{u}}^{g}} \sum_{k=1}^{N_{u} / g} \mathcal{I}\left(\mathcal{S}_{k}\right) .
$$

The proposed GUGA algorithm is an iterative greedy algorithm. Let the set of users which have not been assigned to any group till the end of the $k$-th iteration, be denoted by $\mathbb{V}^{(k)} \subset \mathcal{S}$. In the $(k+1)$-th iteration, a subset of $\mathbb{V}^{(k)}$ containing $g$ users is chosen to be the $(k+1)$-th group of users. Let $\mathbb{E}^{(k)}$ denote the set of all possible ordered subsets of $\mathbb{V}^{(k)}$ of size $g$. That is

$$
\mathbb{E}^{(k)} \triangleq\left\{s \subset \mathbb{V}^{(k)}|| s \mid=g\right\} .
$$

Starting with the $k=0$-th iteration the set $\mathbb{V}^{(0)}=\mathcal{S}$ (i.e., since no user has been grouped so far) and $\mathbb{E}^{(0)}$ is the set of all possible ordered subsets of $\mathcal{S}$ of size $g$. In the $(k+1)$-th iteration, the proposed algorithm finds the group of $g$-users in $\mathbb{E}^{(k)}$ having the maximum rate. This group is then chosen to be the $(k+1)$-th group of users i.e.

$\tilde{\mathcal{S}}_{k+1}=\left\{\mathcal{U}_{(k+1)_{1}}, \mathcal{U}_{(k+1)_{2}}, \cdots, \mathcal{U}_{(k+1)_{g}}\right\} \triangleq \arg \max _{s \in \mathbb{E}^{(k)}} \mathcal{I}(s)$,

where $\mathcal{I}($.$) is given by (36). Let \mathcal{T}^{(k+1)} \subset \mathbb{E}^{(k)}$ be the set of groups of size $g$ having at least one user in the set $\tilde{\mathcal{S}}_{k+1}$. That is

$$
\mathcal{T}^{(k+1)} \triangleq\left\{s \mid s \in \mathbb{E}^{(k)} \text { and } \mathcal{U}_{(k+1)_{j}} \in s \text { for some } j\right\},
$$

where $\mathcal{U}_{(k+1)_{j}}$ is the $j$-th user in the ordered set $\tilde{\mathcal{S}}_{k+1}$. Since the users $\mathcal{U}_{(k+1)_{j}}, j=1,2, \ldots, g$ have been assigned to the $(k+1)$-th group $\tilde{\mathcal{S}}_{k+1}$, at the end of the $(k+1)$-th iteration these users are therefore removed from $\mathbb{V}^{(k)}$, i.e.

$$
\mathbb{V}^{(k+1)}=\mathbb{V}^{(k)} \backslash \tilde{\mathcal{S}}_{k+1},
$$

where " $\backslash$ " denotes the minus/difference operator for sets. From (41) and the definition of $\mathbb{E}^{(k)}$ in (38) we therefore have

$$
\mathbb{E}^{(k+1)}=\mathbb{E}^{(k)} \backslash \mathcal{T}^{(k+1)} .
$$

\footnotetext{
${ }^{14}$ We remind the reader that $\mathbf{R}[k]$ is implicitly dependent on the chosen grouping.
}

The algorithm then moves on to the $(k+2)$-th iteration. Since there are totally $N_{u}$ users and therefore $N_{u} / g$ groups, it is evident that the algorithm terminates after the $N_{g}=\left(N_{u} / g\right)$ th iteration. The proposed grouping of users is then given by

$$
\tilde{\mathcal{P}}=\left\{\tilde{\mathcal{S}}_{1}, \tilde{\mathcal{S}}_{2}, \cdots, \tilde{\mathcal{S}}_{N_{g}}\right\} .
$$

The GUGA algorithm is summarized in the following table.

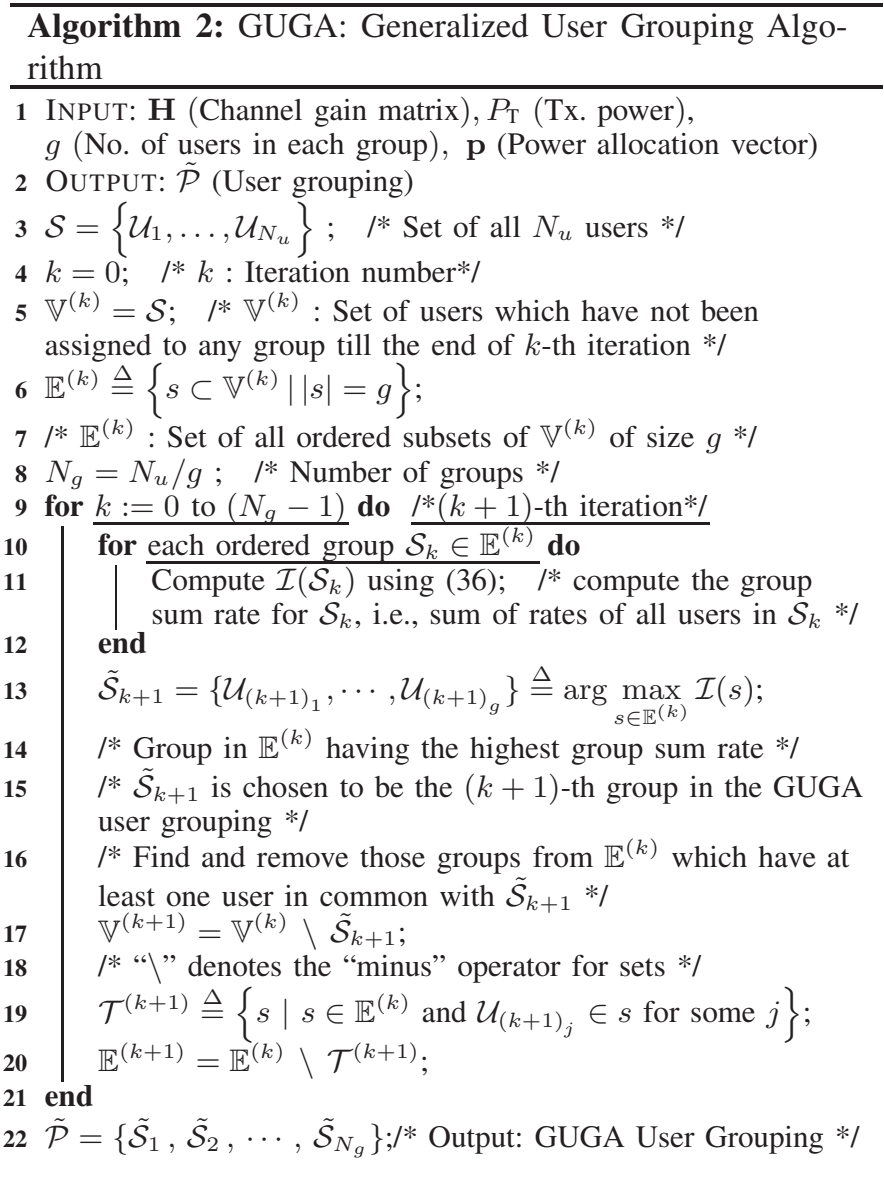

In the following we discuss the complexity of GUGA. The proposed user grouping algorithm (GUGA) needs to initially compute the rate of all possible subsets of $\mathcal{S}$ of size $g$. For a given group, its rate is a function of the corresponding upper triangular matrix representing the effective channel for that group. In Appendix C-A, it is shown that starting with $\left(\mathbf{H} \mathbf{H}^{H}\right)^{-1}$, the complexity of computing the effective upper triangular matrix for a given group is $O\left(g^{3}\right)$. From (36) it then follows that for a given power allocation, computing the rate $\mathcal{I}\left(\mathcal{S}_{k}\right)$ for any arbitrary group of users $\mathcal{S}_{k}$ has a complexity of $O\left(g^{3}\right)$. Since there are $O\left(N_{u}{ }^{g}\right)$ possible ordered groups/subsets of $\mathcal{S}$ of size $g$ (i.e., $\left|\mathbb{E}^{(0)}\right|=O\left(N_{u}{ }^{g}\right)$ ), the complexity of computing the rate of all possible groups/subsets of $\mathcal{S}$ is $O\left(g^{3} N_{u}^{g}\right)$. In the $(k+1)$-th iteration of GUGA, we then find the group of users having the maximum rate among all possible groups in $\mathbb{E}^{(k)}$ (see (39)). The complexity of $N_{g}=N_{u} / g$ iterations of GUGA is therefore $O\left(N_{u}^{g+1}\right)$. Hence we can conclude that the total complexity of GUGA is $O\left(g^{3} N_{u}^{g}\right)+O\left(N_{u}^{g+1}\right)$. 


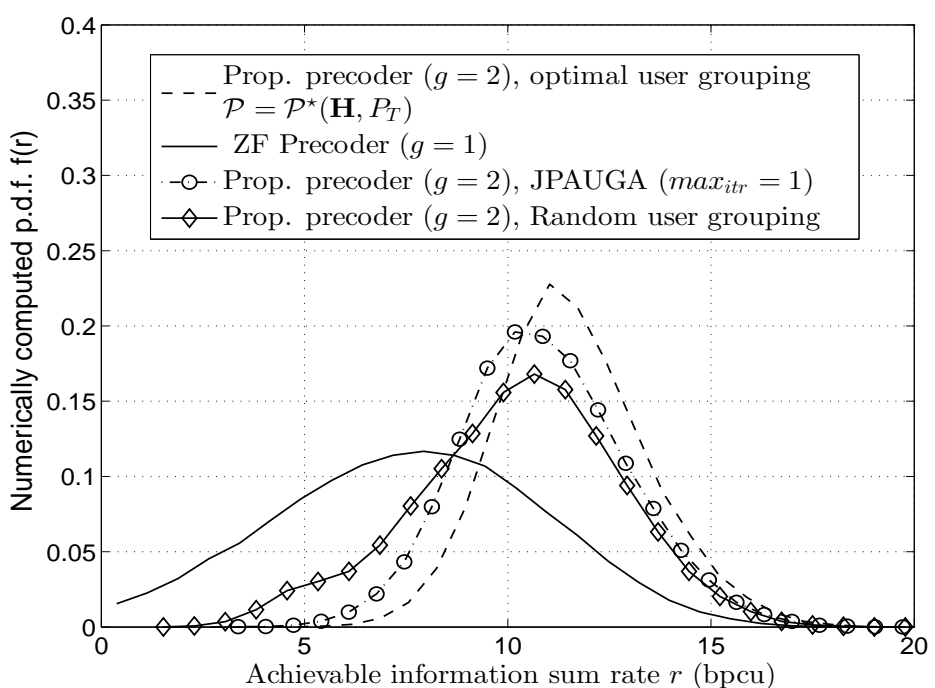

Fig. 3. Numerically computed probability density function (p.d.f.) of the information sum rate achieved by different precoders for a $N_{t}=N_{u}=6$ i.i.d. Rayleigh faded channel with $P_{T}=10 \mathrm{~dB}$.

\section{B. Complexity of the proposed precoder based on JPAUGA}

The whole precoding operation can be broadly divided into two phases. In the first phase, JPAUGA is used to compute the user grouping and the power allocation between users. Then in the second phase, using the JPAUGA user grouping and power allocation, the information for different groups is beamformed in orthogonal directions and information within each group is precoded using DPC.

For the first phase, we need to firstly compute $\left(\mathbf{H H}^{H}\right)^{-1}$ which has a complexity of $O\left(N_{u}^{3}\right)+O\left(N_{u}^{2} N_{t}\right)$. Through numerical simulations we have observed that JPAUGA converges very fast, and few iterations (less than five) are required irrespective of $\left(N_{u}, N_{t}\right)$. The complexity of computing the optimal power allocation for a given user grouping is $O\left(N_{u}^{2}\right)$ (see (23) and (24)). Since each JPAUGA iteration consists of one instance of GUGA followed by waterfilling power allocation, it follows that the total complexity of JPAUGA is $O\left(N_{u}^{3}\right)+O\left(N_{u}^{2} N_{t}\right)+O\left(g^{3} N_{u}^{g}\right)+O\left(N_{u}^{g+1}\right)$.

For the second phase, the complexity of computing the beamforming matrix for a single group is $O\left(g^{3}\right)+O\left(g^{2} N_{u}\right)+$ $O\left(g N_{u} N_{t}\right)+O\left(g^{2} N_{t}\right)$ (see Appendix C-B). Therefore the complexity of computing the beamforming matrices for all the $N_{g}=N_{u} / g$ groups is $O\left(g^{2} N_{u}\right)+O\left(g N_{u}^{2}\right)+O\left(N_{u}^{2} N_{t}\right)+$ $O\left(g N_{u} N_{t}\right)$. The complexity of beamforming the information symbols onto the transmit vector is $O\left(N_{t} N_{u}\right)$ (see (6) and (7)). Additionally, we would also require to perform DPC for $(g-1)$ users in each group. Therefore, the total complexity of the second phase would be $O\left(g^{2} N_{u}\right)+O\left(g N_{u}^{2}\right)+O\left(N_{u}^{2} N_{t}\right)+$ $O\left(g N_{u} N_{t}\right)$ plus the complexity of performing DPC for $N_{g} g$ user MISO-broadcast channels.

The total complexity of the proposed precoder based on JPAUGA (both first and second phase) is therefore $O\left(g^{2} N_{u}\right)+$ $O\left(g N_{u}^{2}\right)+O\left(N_{u}^{3}\right)+O\left(N_{u}^{2} N_{t}\right)+O\left(g N_{u} N_{t}\right)+O\left(g^{3} N_{u}^{g}\right)+$ $O\left(N_{u}^{g+1}\right)$ plus the complexity of performing DPC for $N_{g}$ $g$-user MISO-broadcast channels. Since $N_{t} \geq N_{u} \geq g$ the

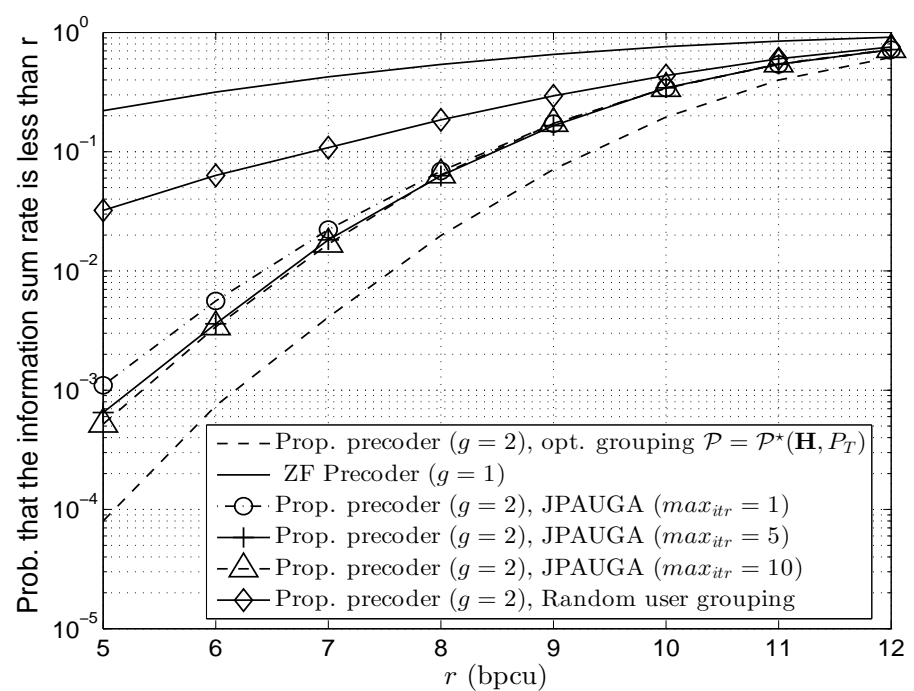

Fig. 4. Probability of the event that the instantaneous sum rate is below a given sum rate $r$. $N_{t}=N_{u}=6$, i.i.d. Rayleigh fading with $P_{T}=10 \mathrm{~dB}$.

total complexity of the proposed precoder is $O\left(N_{u}^{2} N_{t}\right)+$ $O\left(g^{3} N_{u}^{g}\right)+O\left(N_{u}^{g+1}\right)$ plus the complexity of performing DPC. From the expressions for the complexity of the two phases as discussed above, it then follows that the complexity of computing the JPAUGA user grouping and power allocation is completely dominant over the complexity of computing the beamforming matrices for each group and beamforming information to users every channel use.

Remark 2: For small values of $g$ (e.g., $g=2$ ) the effective $g \times g$ lower triangular channel matrix is small enough so that practical near-optimal (i.e., close to DPC) performance achieving schemes can be applied. For example, with $g=2$, due to the lower triangular nature of the effective channel matrix, the first user in each group gets its information symbol interference free, but the second user gets its information symbol along with some interference from the first user's information symbol. However since this interference is already known at the BS, near-optimal interference pre-subtraction can be performed at practical complexity as shown in [8]. Also with $g=2$ the complexity of the proposed JPAUGA and group-wise beamforming is $O\left(N_{u}^{3}\right)+O\left(N_{u}^{2} N_{t}\right)$ i.e., $O\left(N_{u}^{2} N_{t}\right)$ (since $N_{t} \geq N_{u}$ ), which is the same as the complexity of the ZF precoder.

\section{Simulation Results}

In this section we consider an i.i.d. Rayleigh fading channel, i.e., the channel gains $h_{k, i}^{*}$ are i.i.d. $\mathcal{C N}(0,1)$. In Fig. 3 we consider a $N_{t}=N_{u}=6$ i.i.d. Rayleigh fading channel with $P_{T}=10 \mathrm{~dB}$, for which we numerically compute and plot the probability density function (p.d.f.) of the sum rate achieved by the $\mathrm{ZF}$ precoder (i.e., $r=C_{Z F}\left(\mathbf{H}, P_{T}\right)$ ), the proposed user grouping precoder with optimal user pairing (i.e., $r=$ $r^{\star}\left(\mathbf{H}, P_{T}\right)$ with $\left.g=2\right)$, the proposed precoder with random user pairing ${ }^{15}$, and the proposed precoder with JPAUGA $(g=$

\footnotetext{
${ }^{15}$ Pairs of users $(g=2)$ being chosen randomly independent of the channel realization, followed by optimal waterfilling power allocation for the randomly chosen user pairing.
} 


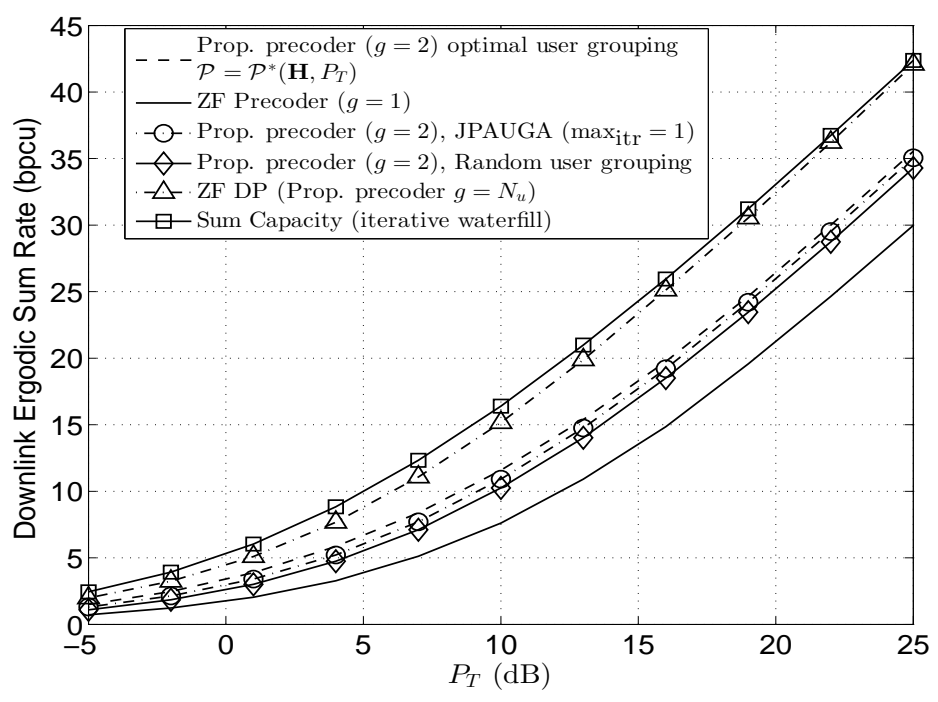

Fig. 5. Ergodic sum rate versus $P_{T}(\mathrm{~dB}) . N_{t}=N_{u}=6$ and i.i.d. Rayleigh fading.

2 and $\left.\max _{i t r}=1\right)$. The achievable sum rate for each precoder is random due to the random channel gains. It can be observed from the figure that the probability of the sum rate assuming small values (compared to the mean value, i.e., ergodic rate) is much higher for the ZF precoder than for the proposed user grouping based precoders. For example, the sum rate of the ZF precoder is less than 6 bpcu with a probability of 0.2 (i.e., for every fifth channel realization on an average), whereas the sum rate achieved by the proposed precoder based on JPAUGA user pairing $\left(\max _{i t r}=1\right)$ falls below 6 bpcu with a probability less than 0.01 (i.e., one in hundred channel realizations). Therefore, in a way the proposed user grouping based precoders improve the conditioning of the channel.

We also represent the numerical data collected for Fig. 3, in terms of the probability that a given precoding scheme achieves an instantaneous information sum rate less than some specified rate $r$. This is shown in Fig. 4, where it can be clearly seen that for a given fixed rate $r$, compared to the ZF precoder the proposed precoders (with $g=2$ ) have a significantly lower probability of the event that the instantaneous information sum rate falls below $r$. For any precoder let us define its critical rate $r$ to be such that the probability that its instantaneous information sum rate falls below $r$ bpcu equals $1 \times 10^{-3}$. It can be observed that the critical value of $r$ for the proposed precoder with JPAUGA based user grouping (only one iteration) is 5 bpcu which is only about 1 bpcu less than the critical rate of the proposed precoder with optimal user grouping. Numerical simulations reveal that the critical rate of the ZF precoder is only about $0.1 \mathrm{bpcu}$, and therefore using the proposed precoder based on JPAUGA user grouping results in a 50 fold increase in the critical rate when compared to the ZF precoder. It is noted that the proposed precoder based on JPAUGA user pairing achieves this performance improvement at a complexity similar to the ZF precoder (see Remark 2 in Section V-B).

In Fig. 4, we also plot the curves for the proposed precoder

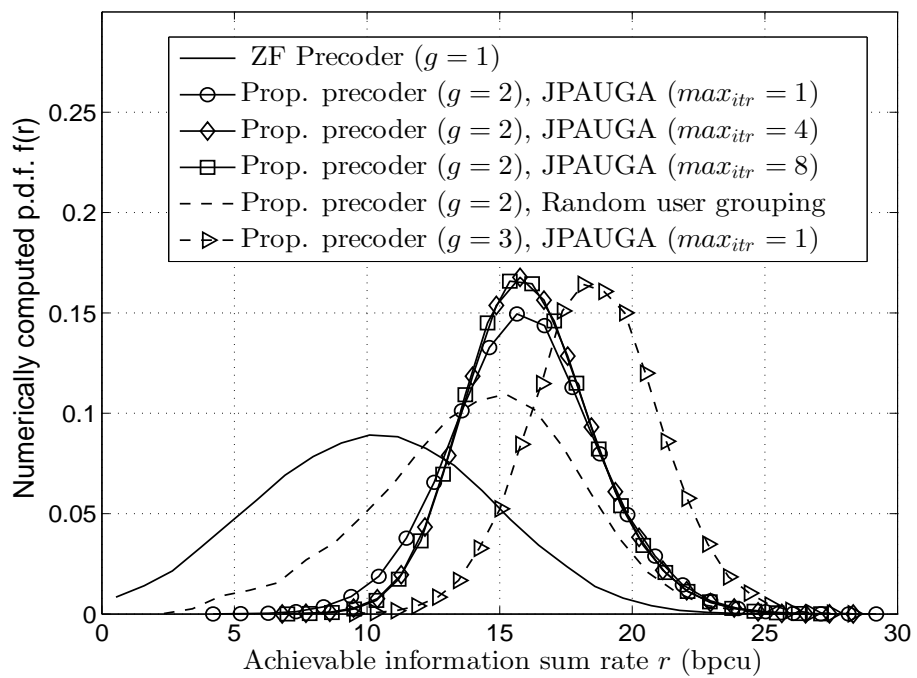

Fig. 6. Numerically computed probability density function (p.d.f.) of the information sum rate achieved by different precoders for a $N_{t}=N_{u}=12$ i.i.d. Rayleigh fading channel with $P_{T}=10 \mathrm{~dB}$.

based on JPAUGA user grouping $(g=2)$, for $\max _{i t r}=5$ and $\max _{i t r}=10$. It can be seen that the performance improves with increasing number of iterations. However this improvement in performance is small relative to the improvement achieved by switching from random user grouping to optimal user grouping. This also supports the comment made in Section V-B, on the fast convergence of JPAUGA.

In Fig. 5 we investigate the ergodic (average) information sum rate achieved by the various precoding schemes as a function of increasing $P_{T}$, for a fixed $N_{t}=N_{u}=6$ and i.i.d. Rayleigh fading. It is observed that for a given $P_{T}$ the proposed user grouping based precoders $(g=2)$ achieve a larger ergodic sum rate than the ZF precoder. For small $P_{T}=5 \mathrm{~dB}$, the proposed precoder based on JPAUGA user grouping $\left(\max _{\mathrm{itr}}=1\right)$ achieves a sum rate of 6 bpcu which is about 1.5 times the sum rate achieved by the $\mathrm{ZF}$ precoder. For larger $P_{T}$, it appears that the ratio between the sum rate achieved by the proposed precoders and the ZF precoder approaches one. However, to achieve a given desired sum rate, the extra total transmit power required by the ZF precoder (when compared to the proposed precoders) is observed to be roughly $3 \mathrm{~dB}$ over a large range of achievable sum rates ( 5 bpcu to $30 \mathrm{bpcu}$ ). For the proposed precoder, it is also observed that even random user grouping achieves an ergodic sum rate performance close to that achieved by the optimal user grouping and the JPAUGA user grouping. The subtle but important point however is that, though the ergodic sum rates are roughly the same, from Fig. 4 it is clear that the probability of the sum rate being small is larger when random user grouping is used.

In Fig. 6, we plot the numerically estimated p.d.f. of the achievable sum rate for $N_{t}=N_{u}=12$. We are unable to plot the p.d.f. of the sum rate achieved by the proposed precoder with optimal user grouping due to its prohibitive complexity (with $g=2$ the number of possible groupings is only 120 when $N_{u}=6$, but which increases to 665280 when $N_{u}=12$ ). 


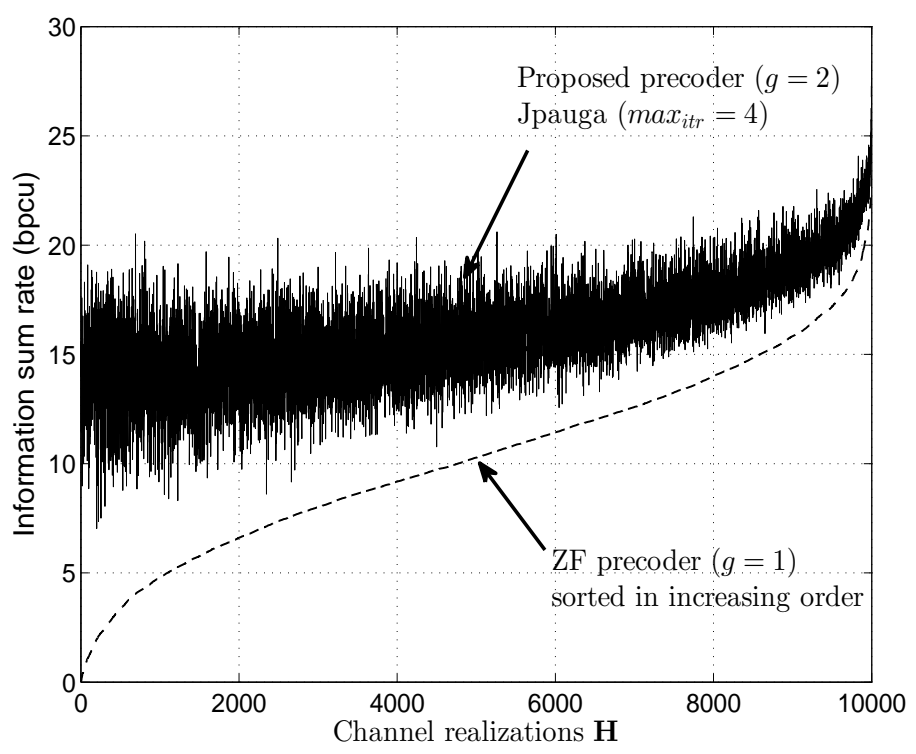

Fig. 7. Sum rate of the proposed precoder with JPAUGA user grouping $\left(g=2, \max _{i t r}=4\right)$ and the $\mathrm{ZF}$ precoder for ten thousand random channel realizations $\left(N_{t}=N_{u}=12\right.$, i.i.d. Rayleigh fading and $\left.P_{T}=10 \mathrm{~dB}\right)$.

From Fig. 6 we can make observations similar to that made in Fig. 3. In Fig. 6 we have also shown the p.d.f. of the proposed user grouping based on JPAUGA user grouping with $g=3$. It is observed that by grouping $g=3$ users the p.d.f. shifts to the right when compared to $g=2$, which implies an even higher ergodic sum rate and an even lower probability of the sum rate being small. This improvement in performance in going from $g=2$ to $g=3$ however comes at the cost of increased complexity (see Section V-B).

In Fig. 7 we plot the achievable sum rate of the proposed precoder (JPAUGA user grouping with $g=2$ and $\max _{i t r}=4$ ) and that of the $\mathrm{ZF}$ precoder for ten thousand random channel realizations $\left(N_{t}=N_{u}=12\right.$, i.i.d. Rayleigh fading and $P_{T}=$ $10 \mathrm{~dB})$. In the plot the realizations have been reordered so that channel realization number 1 is the realization for which the sum rate achieved by the $\mathrm{ZF}$ precoder is the least among the sum rates achieved by the $\mathrm{ZF}$ precoder for all the ten thousand realizations. Channel realization number 2 is the realization for which the sum rate achieved by the $\mathrm{ZF}$ precoder is the second least and so on. ${ }^{16}$ We observe that for ill-conditioned channel realizations where the $\mathrm{ZF}$ precoder achieves small information sum rate, the proposed user grouping based precoder achieves a much better performance. ${ }^{17}$

\section{FUTURE RESEARCH DIRECTIONS}

In this section we briefly discuss two possible ways in which the proposed user grouping precoder could be extended to

\footnotetext{
${ }^{16}$ Since the ordering is based on the sum rate achieved by the $\mathrm{ZF}$ precoder, it is clear that the sum rate versus the ordered channel realization number curve is smooth and monotonically increasing for the ZF precoder, whereas that for the proposed precoder is fluctuating.

${ }^{17}$ For channel realization indices between 1 and 400 the ZF precoder achieves a sum rate less than $3 \mathrm{bpcu}$. For these same channel realizations the proposed user grouping based precoder always achieves a sum rate greater than 7 bpcu.
}

MIMO broadcast channels where users can have more than one receive antenna.

The first possible extension is to transform the MIMO broadcast channel into a MISO broadcast channel and then use the proposed grouping algorithm on the transformed MISO broadcast channel. For this transformation, we consider each physical antenna of each user as a separate "user". Therefore, if there are $N_{u}$ users having $N_{r}$ antennas each, then we have converted the original MIMO broadcast channel into a MISO broadcast channel with $N_{u} N_{r}$ autonomous single antenna "users". With this extension, it is possible that different antennas of a user could be assigned to different groups. The advantage of this extension is that the signal received at any antenna of a user is free of interference from information signals communicated to the other antennas of that user. Therefore, no extra receiver processing is required.

In the second possible extension we treat all physical antennas of a user as one entity, and if two users are grouped together then so are all their physical antennas. While forming the group-wise matrices $\mathbf{G}[i], i=1,2, \ldots, N_{g}$ and the complementary matrices $\mathbf{H}[i], i=1,2, \ldots, N_{g}$, it must be ensured that channel gain vectors of antennas of a given user are placed in consecutive rows of the same matrix. Intergroup interference can be cancelled in exactly the same way as discussed in Section IV-A for MISO broadcast channels. This ensures that the per-group effective channel gain matrices $\mathbf{B}[i], i=1,2, \ldots, N_{g}$ are lower triangular with the effective channel gain vectors for all antennas of a given user lying in consecutive rows of the same effective channel matrix. Pre-cancellation of intra-group interference using dirty paper coding (DPC) can be done in a successive manner as has been proposed in Section IV-B. However, for a user having more than one receive antenna, vector DPC must be performed as compared to scalar DPC for users having only one receive antenna.

It is not clear as to which of the above two extensions would achieve a higher sum rate. This is an interesting study which could be taken up as an extension to the work presented in this paper.

\section{CONCLUSIONS}

In this paper, we proposed a precoding scheme in which users are grouped together in small groups of size $g$. Multiuser beamforming is done in such a way that only inter-group interference is cancelled, resulting in $N_{u} / g$ parallel noninterfering $g \times g$ Gaussian MISO broadcast channels, one such channel for each group. Due to the lower triangular structure of the equivalent $g \times g$ broadcast channel for each group, successive DPC can be used to pre-cancel the intra-group interference within each group. This method of precoding is shown to achieve a significantly better performance than the ZF precoder, especially when the channel is ill-conditioned. The sum rate achieved by the proposed precoder is also shown to be sensitive towards the chosen user grouping, and therefore a novel low-complexity joint power allocation and user grouping algorithm (JPAUGA) is proposed. 


\section{APPENDIX A}

PROOF OF THEOREM 4.1

For a given $\left(\mathbf{H}, P_{T}, \mathcal{P}\right)$, from (22) it is clear that

$$
r\left(\mathbf{H}, P_{T}, \mathcal{P}\right) \geq r\left(\mathbf{H}, P_{T}, \mathcal{P}, \mathbf{p}^{*}\right),
$$

since the optimal power allocation for the ZF precoder i.e., $\mathbf{p}^{*}$ (see (26)) is not necessarily the optimal power allocation for the proposed precoder with $g \geq 2$. Hence in order to prove (28) for any $\left(\mathbf{H}, P_{T}, \mathcal{P}\right)$ with the user grouping $\mathcal{P}$ having groups of size $g \geq 2$, it suffices to show that $r\left(\mathbf{H}, P_{T}, \mathcal{P}, \mathbf{p}^{*}\right) \geq$ $C_{\mathrm{ZF}}\left(\mathbf{H}, P_{T}\right)$, i.e.

$$
\sum_{k=1}^{N_{u} / g} \sum_{j=1}^{g} \log _{2}\left(1+p_{k_{j}}^{*} \mathbf{R}[k]_{(j, j)}^{2}\right) \geq C_{\mathrm{ZF}}\left(\mathbf{H}, P_{T}\right) .
$$

Here, in the L.H.S. we have used the expression for $r\left(\mathbf{H}, P_{T}, \mathcal{P}, \mathbf{p}\right)$ from (21). In the following we will show that for any arbitrary $\mathcal{P}$

$$
\mathbf{R}[k]_{(j, j)}^{2} \geq \frac{1}{\left[\left(\mathbf{H H}^{H}\right)^{-1}\right]_{\left(k_{j}, k_{j}\right)}} .
$$

This is sufficient to prove (28) because combining (46) and (25), we get (45).

Since $\mathbf{R}[k]$ is the upper triangular matrix in the QR-type decomposition of $\mathbf{F}[k]$, we next examine the columns of $\mathbf{F}[k]=\mathbf{P}[k] \mathbf{G}[k]^{H}$. The $j$-th column of $\mathbf{G}[k]^{H}$ is nothing but the complex conjugate of the channel vector of the user $\mathcal{U}_{k_{j}}$. We firstly note that, the $j$-th column of $\mathbf{F}[k]$ is the projection of the channel vector of user $\mathcal{U}_{k_{j}}$ onto $\mathcal{H}_{k}^{\perp}$, i.e., the space orthogonal to the space spanned by the channel vectors of users not in the $k$-th group. Remember that for user $\mathcal{U}_{k_{j}}, \mathcal{C}_{k_{j}} \subset \mathbb{C}^{N_{t}}$ is the space of vectors orthogonal to the space spanned by the rows of $\mathbf{H}[k]$ and the rows of the previous $(j-1)$ users in the $k$-th group (i.e., $\left.\mathbf{h}_{k_{1}}^{H}, \mathbf{h}_{k_{2}}^{H}, \cdots, \mathbf{h}_{k_{(j-1)}}^{H}\right)$. Since $\mathrm{QR}$-decomposition is essentially a Gram-Schmidt orthogonalization procedure, $\mathbf{R}[k]_{(j, j)}$ is nothing but the Euclidean length of the projection of the channel vector of user $\mathcal{U}_{k_{j}}$ (i.e., $\mathbf{h}_{k_{j}}^{H}$ ) onto the space $\mathcal{C}_{k_{j}}$.

In the case of ZF precoding, each group has only one user, and is therefore a special case of the proposed user grouping scheme. For the user $\mathcal{U}_{k_{j}}$, with $\mathrm{ZF}$ precoding, the effective channel gain is therefore the Euclidean length of the projection of $\mathbf{h}_{k_{j}}^{H}$ onto the space orthogonal to the space spanned by the channel vectors of the remaining $\left(N_{u}-1\right)$ users. In Section III, for user $\mathcal{U}_{k_{j}}$, we had used $\mathcal{H}_{k_{j}}^{\perp}$ to denote the space orthogonal to the space spanned by the channel vectors of the remaining $\left(N_{u}-1\right)$ users. From the definition of the space $\mathcal{C}_{k_{j}}$, it follows that $\mathcal{H}_{k_{j}}^{\perp}$ is a subspace of $\mathcal{C}_{k_{j}}$.

$$
\mathcal{H}_{k_{j}}^{\perp} \subset \mathcal{C}_{k_{j}} .
$$

We next show that the Euclidean length of the projection of $\mathbf{h}_{k_{j}}^{H}$ onto $\mathcal{H}_{k_{j}}^{\perp}$ is equal to $1 / \sqrt{\left[\left(\mathbf{H H}^{H}\right)^{-1}\right]_{k_{j}, k_{j}}}$. Consider a row permutation matrix $\mathbf{T} \in \mathbb{C}^{N_{u} \times N_{u}}$, which swaps the $k_{j}$-th row with the first row of any matrix with $N_{u}$ rows. Then the matrix TH $\in \mathbb{C}^{N_{u} \times N_{t}}$ has the following structure

$$
\mathbf{T H}=\left[\begin{array}{c}
\mathbf{h}_{k_{j}}^{H} \\
\tilde{\mathbf{H}}
\end{array}\right],
$$

where $\tilde{\mathbf{H}}=\left(\mathbf{h}_{2}, \mathbf{h}_{3}, \cdots, \mathbf{h}_{k_{(j-1)}}, \mathbf{h}_{1}, \mathbf{h}_{k_{(j+1)}}, \cdots \mathbf{h}_{N_{u}}\right)^{H}$ is a sub-matrix of $\mathbf{H}$ containing all the rows of $\mathbf{H}$ except $\mathbf{h}_{k_{j}}^{H}$, and with $\mathbf{h}_{1}^{H}$ replacing $\mathbf{h}_{k_{j}}^{H}$ in the $k_{j}$-th row. Here we also note that, $\mathcal{H}_{k_{j}}^{\perp}$ is the space of vectors orthogonal to the rows of $\tilde{\mathbf{H}}$. The Euclidean length of the projection of $\mathbf{h}_{k_{j}}^{H}$ onto the space $\mathcal{H}_{k_{j}}^{\perp}$ is given by

$$
\begin{aligned}
c_{k_{j}} & =\left\|\left(\mathbf{I}_{N_{t}}-\tilde{\mathbf{H}}^{H}\left(\tilde{\mathbf{H}} \tilde{\mathbf{H}}^{H}\right)^{-1} \tilde{\mathbf{H}}\right) \mathbf{h}_{k_{j}}\right\| \\
& =\sqrt{\mathbf{h}_{k_{j}}^{H} \mathbf{h}_{k_{j}}-\mathbf{h}_{k_{j}}^{H} \tilde{\mathbf{H}}^{H}\left(\tilde{\mathbf{H}} \tilde{\mathbf{H}}^{H}\right)^{-1} \tilde{\mathbf{H}} \mathbf{h}_{k_{j}}} .
\end{aligned}
$$

We now consider the matrix $\mathbf{T} \mathbf{H} \mathbf{H}^{H} \mathbf{T}^{H} \in \mathbb{C}^{N_{u} \times N_{u}}$ which has the following structure:

$$
\mathbf{T H H}^{H} \mathbf{T}^{H}=\left[\begin{array}{cc}
\mathbf{h}_{k_{j}}^{H} \mathbf{h}_{k_{j}} & \mathbf{h}_{k_{j}}^{H} \tilde{\mathbf{H}}^{H} \\
\tilde{\mathbf{H}} \mathbf{h}_{k_{j}} & \tilde{\mathbf{H}} \tilde{\mathbf{H}}^{H}
\end{array}\right],
$$

The inverse of the block partitioned matrix in (50) is given by

$$
\begin{aligned}
& \left(\mathbf{T H H}{ }^{H} \mathbf{T}^{H}\right)^{-1} \\
& \quad=\left[\begin{array}{cc}
\left(\mathbf{h}_{k_{j}}^{H} \mathbf{h}_{k_{j}}-\mathbf{h}_{k_{j}}^{H} \tilde{\mathbf{H}}^{H}\left(\tilde{\mathbf{H}} \tilde{\mathbf{H}}^{H}\right)^{-1} \tilde{\mathbf{H}} \mathbf{h}_{k_{j}}\right)^{-1} & \mathbf{Y} \\
\mathbf{Z} & \mathbf{W}
\end{array}\right],
\end{aligned}
$$

with appropriate block matrices $\mathbf{Y}, \mathbf{Z}$ and $\mathbf{W}$. Here we have used the result that for any square full rank block partitioned matrix $\mathbf{V}$, of the form

$$
\mathbf{V}=\left[\begin{array}{ll}
\mathbf{A} & \mathbf{B} \\
\mathbf{C} & \mathbf{D}
\end{array}\right]
$$

the inverse is given by [26]

$\mathbf{V}^{-1}=\left[\begin{array}{cc}\left(\mathbf{A}-\mathbf{B D}^{-1} \mathbf{C}\right)^{-1} & -\mathbf{A}^{-1} \mathbf{B}\left(\mathbf{D}-\mathbf{C A}^{-1} \mathbf{B}\right)^{-1} \\ -\mathbf{D}^{-1} \mathbf{C}\left(\mathbf{A}-\mathbf{B D}^{-1} \mathbf{C}\right)^{-1} & \left(\mathbf{D}-\mathbf{C A}^{-1} \mathbf{B}\right)^{-1}\end{array}\right]$.

From (49) and (51) it is clear that the squared Euclidean length of the projection of $\mathbf{h}_{k_{j}}^{H}$ onto the space orthogonal to the rows of $\tilde{\mathbf{H}}$ is simply the inverse of the $(1,1)$ entry of the matrix $\left(\mathbf{T H H} \mathbf{H}^{H} \mathbf{T}^{H}\right)^{-1}$, i.e.

$$
c_{k_{j}}^{2}=\frac{1}{\left[\left(\mathbf{T H H}^{H} \mathbf{T}^{H}\right)^{-1}\right]_{(1,1)}} .
$$

Since $\mathbf{T}$ swaps the $k_{j}$-th and the first row of $\mathbf{H}$, it follows that

$$
\begin{aligned}
{\left[\left(\mathbf{T H H}{ }^{H} \mathbf{T}^{H}\right)^{-1}\right]_{(1,1)} } & =\left[\mathbf{T}\left(\mathbf{H} H^{H}\right)^{-1} \mathbf{T}^{H}\right]_{(1,1)} \\
& =\left[\left(\mathbf{H} \mathbf{H}^{H}\right)^{-1}\right]_{\left(k_{j}, k_{j}\right)} .
\end{aligned}
$$

Combining (54) and (55), we have

$$
c_{k_{j}}=\frac{1}{\sqrt{\left[\left(\mathbf{H H}^{H}\right)^{-1}\right]\left(k_{j}, k_{j}\right)}} .
$$

For the proposed user grouping algorithm, for any arbitrary grouping, the projection of the channel vector of user $\mathcal{U}_{k_{j}}$ (i.e., $\mathbf{h}_{k_{j}}^{H}$ ) onto the subspace $\mathcal{C}_{k_{j}}$ is equal to $\mathbf{R}[k]_{(j, j)}$. From (56), the projection of $\mathbf{h}_{k_{j}}^{H}$ onto the subspace $\mathcal{H}_{k_{j}}^{\perp}$ is equal to $1 / \sqrt{\left[\left(\mathbf{H H}^{H}\right)^{-1}\right]_{\left(k_{j}, k_{j}\right)}}$. From (47), it follows that $\mathcal{H}_{k_{j}}^{\perp}$ is a subspace of $\mathcal{C}_{k_{j}}$, which implies that the projection of $\mathbf{h}_{k_{j}}^{H}$ 
onto $\mathcal{H}_{k_{j}}^{\perp}$ has a smaller Euclidean length than its projection on $\mathcal{C}_{k_{j}}{ }^{18}$. From the above arguments,

$$
\mathbf{R}[k]_{(j, j)} \geq \frac{1}{\sqrt{\left[\left(\mathbf{H H}^{H}\right)^{-1}\right]_{\left(k_{j}, k_{j}\right)}}},
$$

which proves (46) and subsequently (45).

\section{APPENDIX B}

\section{PROOF OF LEMMA 1}

Towards proving Lemma 1, we firstly observe that the $\mathrm{ZF}$ precoder is a special case of the proposed precoder with $g=1$. Further it is trivial to show that for the proposed precoder with $g=2$, out of the two users in any given pair, one user (to be precise, user $\mathcal{U}_{k_{2}}$ for the $k$-th pair) has exactly the same channel gain as it would have had if $\mathrm{ZF}$ precoding were to be used. The "other" user in the pair (i.e., user $\mathcal{U}_{k_{1}}$ for the $k$-th pair) has a larger effective channel gain magnitude compared to its effective channel gain if the $\mathrm{ZF}$ precoder were to be used. ${ }^{19}$ For notational simplicity, let the effective channel gain of the user $\mathcal{U}_{k_{1}}$ be denoted by $a_{k}(\mathbf{H})$ when precoding with $g=2$ (i.e, the proposed precoder with users grouped in pairs) and by $b_{k}(\mathbf{H})$ when precoding with the ZF precoder (i.e., $g=$ $1)$. We are interested in evaluating the difference in the ergodic sum rates achieved by the proposed precoder when precoding with $g=2$ and with $g=1$ respectively. Since user $\mathcal{U}_{k_{2}}$ of the $k$-th pair has the same rate irrespective of whether $g=1$ or $g=2$, the difference in the ergodic sum rates is given by

$$
\begin{aligned}
d\left(P_{T}, N_{u}\right)=\sum_{k=1}^{N_{u} / 2}(\mathbb{E} & {\left[\log _{2}\left(1+\frac{P_{T}}{N_{u}} a_{k}(\mathbf{H})^{2}\right)\right] } \\
& \left.-\mathbb{E}\left[\log _{2}\left(1+\frac{P_{T}}{N_{u}} b_{k}(\mathbf{H})^{2}\right)\right]\right) .
\end{aligned}
$$

The expectation in (58) is over the distribution of $\mathbf{H}$. Further, due to i.i.d. fading statistics and the fact that the pairing of users is independent of the channel realization, it turns out that the $N_{u} / 2$ random variables $a_{k}(\mathbf{H}), k=1,2, \cdots, N_{u} / 2$ are identically distributed, and a similar thing is true for $b_{k}(\mathbf{H}), k=1,2, \cdots, N_{u} / 2$. Therefore, (58) can be written as

$$
\begin{aligned}
& d\left(P_{T}, N_{u}\right)=\frac{N_{u}}{2}(\mathbb{E} {\left[\log _{2}\left(1+\frac{P_{T}}{N_{u}} a_{k}(\mathbf{H})^{2}\right)\right] } \\
&\left.\quad-\mathbb{E}\left[\log _{2}\left(1+\frac{P_{T}}{N_{u}} b_{k}(\mathbf{H})^{2}\right)\right]\right) .
\end{aligned}
$$

With i.i.d. Rayleigh fading, twice the squared Euclidean length of the projection of the channel vector of a given user onto the space orthogonal to the range space spanned by the channel vectors of $N_{u}-g$ out of the remaining $N_{u}-1$ users is $\chi^{2}$ distributed with $2\left(N_{t}-N_{u}+g\right)$ degrees of freedom. This result follows immediately from the distribution of the diagonal elements of the upper triangular matrix in the $\mathrm{QR}$

\footnotetext{
${ }^{18}$ The fact used here is that, the Euclidean length of the projection of any vector onto a subspace $\mathcal{B} \subset \mathcal{G}$ is smaller than its projection onto the original space $\mathcal{G}$. This can be proved using elementary linear algebra.

${ }^{19}$ This follows from the proof of Theorem 4.1.
}

factorization of the i.i.d. Gaussian matrix $\mathbf{H}^{H}$ [23]. Further, $a_{k}(\mathbf{H})$ and $b_{k}(\mathbf{H})$ are nothing but the Euclidean length of the projection of $\mathbf{h}_{k_{1}}^{H}$ onto the subspaces $\mathcal{C}_{k_{1}}$ and $\mathcal{H}_{k_{1}}^{\perp}$ respectively. It can therefore be concluded that with $N_{t}=N_{u}, 2 a_{k}(\mathbf{H})^{2}$ and $2 b_{k}(\mathbf{H})^{2}$ are $\chi^{2}$ distributed with 4 and 2 degrees of freedom respectively. Therefore, (59) can be simplified to

$d\left(P_{T}, N_{u}\right)=\frac{N_{u}}{2} \log _{2}(e) \int_{0}^{\infty}(x-1) e^{-x} \log \left(1+\frac{P_{T}}{N_{u}} x\right) d x$.

After some algebraic manipulations, we have

$$
d\left(P_{T}, N_{u}\right)=\frac{N_{u}}{2} \log _{2}(e)\left(1-\frac{N_{u}}{P_{T}} e^{\frac{N_{u}}{P_{T}}} E_{1}\left(\frac{N_{u}}{P_{T}}\right)\right)
$$

where $E_{1}(z) \triangleq \int_{z}^{\infty} e^{-t} / t d t$ is the exponential integral. For $z>0$ it is known that [24]

$$
\frac{1}{2} \log \left(1+\frac{2}{z}\right)<e^{z} E_{1}(z)<\log \left(1+\frac{1}{z}\right) .
$$

Using (62) in (61) with $z=N_{u} / P_{T}$, we have

$$
\begin{aligned}
\frac{N_{u}}{2} \log _{2}(e)( & \left.-\frac{N_{u}}{P_{T}} \log \left(1+\frac{P_{T}}{N_{u}}\right)\right)<d\left(P_{T}, N_{u}\right) \\
& <\frac{N_{u}}{2} \log _{2}(e)\left(1-\frac{N_{u}}{2 P_{T}} \log \left(1+\frac{2 P_{T}}{N_{u}}\right)\right),
\end{aligned}
$$

which proves the theorem.

\section{APPENDIX C}

EFFICIENT COMPUTATION OF THE EFFECTIVE CHANNEL MATRIX R $[k]^{H}$ AND THE BEAMFORMING MATRIX $\mathbf{Q}[k]$ FOR ANY ARBITRARY ORDERED GROUP

$$
\mathcal{S}_{k}=\left\{\mathcal{U}_{k_{1}}, \mathcal{U}_{k_{2}}, \cdots, \mathcal{U}_{k_{g}}\right\}
$$

Since the proposed JPAUGA needs to compute the rate $\mathcal{I}(\cdot)$ for all possible groups of $g$-users, we propose an efficient method to compute $\mathbf{R}[k]$ for any arbitrary group of users. This is discussed in Section C-A. Once the user grouping and power allocation is decided by JPAUGA, the group-wise beamforming matrices $\mathbf{Q}[k], k=1,2, \ldots, N_{g}$ need to be computed. From (13) we know that $\mathbf{F}[k]=\mathbf{Q}[k] \mathbf{R}[k]$, and therefore $\mathbf{Q}[k]$ can be computed from the QR decomposition of $\mathbf{F}[k]$. Efficient computation of $\mathbf{F}[k]$ and its QR-decomposition is discussed in Section C-B.

\section{A. Computation of $\mathbf{R}[k]$ from $\left(\mathbf{H} \mathbf{H}^{H}\right)^{-1}$}

For the ordered group of users $\mathcal{S}_{k}=\left\{\mathcal{U}_{k_{1}}, \mathcal{U}_{k_{2}}, \cdots, \mathcal{U}_{k_{g}}\right\}$, consider the row permutation matrix $\mathbf{T}[k]$ such that

$$
\mathbf{T}[k] \mathbf{H}=\left[\begin{array}{c}
\mathbf{G}[k] \\
\mathbf{H}[k]
\end{array}\right]
$$

Let $\mathbf{A}[k] \in \mathbb{C}^{N_{u} \times g}$ denote the matrix consisting of only the first $g$ columns of $\left(\mathbf{T}[k] \mathbf{H} \mathbf{H}^{H} \mathbf{T}[k]^{H}\right)^{-1}$. Using the expression for the inverse of block partitioned matrices in (53), $\mathbf{A}[k]$ is given by (65) (shown in the top of the next page). Next, we make an important observation that $\mathbf{F}[k]^{H} \mathbf{F}[k]$ is nothing but the inverse of the upper $g \times g$ sub-matrix of $\mathbf{A}[k]$ (see (66)). In (66), step (a) follows from the fact that $\mathbf{F}[k]=\mathbf{P}[k] \mathbf{G}[k]^{H}$ 


$$
\mathbf{A}[k]=\left[\begin{array}{c}
\left(\mathbf{G}[k] \mathbf{G}[k]^{H}-\mathbf{G}[k] \mathbf{H}[k]^{H}\left(\mathbf{H}[k] \mathbf{H}[k]^{H}\right)^{-1} \mathbf{H}[k] \mathbf{G}[k]^{H}\right)^{-1} \\
-\left(\mathbf{H}[k] \mathbf{H}[k]^{H}\right)^{-1} \mathbf{H}[k] \mathbf{G}[k]^{H}\left(\mathbf{G}[k] \mathbf{G}[k]^{H}-\mathbf{G}[k] \mathbf{H}[k]^{H}\left(\mathbf{H}[k] \mathbf{H}[k]^{H}\right)^{-1} \mathbf{H}[k] \mathbf{G}[k]^{H}\right)^{-1}
\end{array}\right] .
$$

$$
\begin{aligned}
\mathbf{F}[k]^{H} \mathbf{F}[k] \stackrel{(\text { (a) }}{=} \mathbf{G}[k] \mathbf{P}[k] \mathbf{G}[k]^{H}=\left(\mathbf{G}[k] \mathbf{G}[k]^{H}-\mathbf{G}[k] \mathbf{H}[k]^{H}\left(\mathbf{H}[k] \mathbf{H}[k]^{H}\right)^{-1} \mathbf{H}[k] \mathbf{G}[k]^{H}\right) \\
\stackrel{(b)}{=} \text { inverse of the upper } g \times g \text { sub-matrix of } \mathbf{A}[k] .
\end{aligned}
$$

$$
\begin{array}{r}
\mathbf{H}^{H} \mathbf{T}[k]^{H}\left(\mathbf{A}[k]\left(\mathbf{G}[k] \mathbf{G}[k]^{H}-\mathbf{G}[k] \mathbf{H}[k]^{H}\left(\mathbf{H}[k] \mathbf{H}[k]^{H}\right)^{-1} \mathbf{H}[k] \mathbf{G}[k]^{H}\right)\right)=\left[\mathbf{G}[k]^{H} \mathbf{H}[k]^{H}\right]\left[\begin{array}{c}
\mathbf{I}_{g} \\
-\left(\mathbf{H}[k] \mathbf{H}[k]^{H}\right)^{-1} \mathbf{H}[k] \mathbf{G}[k]^{H}
\end{array}\right] \\
=\mathbf{G}[k]^{H}-\mathbf{H}[k]^{H}\left(\mathbf{H}[k] \mathbf{H}[k]^{H}\right)^{-1} \mathbf{H}[k] \mathbf{G}[k]^{H}=\mathbf{P}[k] \mathbf{G}[k]^{H}=\mathbf{F}[k] .(67)
\end{array}
$$

and step (b) follows from ((65)). From (13) we know that $\mathbf{F}[k]^{H} \mathbf{F}[k]=\mathbf{R}[k]^{H} \mathbf{R}[k]$ and therefore $\mathbf{R}[k]$ can be computed from the Cholesky factorization [26] of the inverse of the upper $g \times g$ sub-matrix of $\mathbf{A}[k]$ (see ((66))). This Cholesky factorization has a complexity of $O\left(g^{3}\right)$. In the following we therefore discuss the computation of the upper $g \times g$ sub-matrix of $\mathbf{A}[k]$.

We make an important note here that, even though $\mathbf{A}[k]$ consists of the first $g$ columns of $\left(\mathbf{T}[k] \mathbf{H} \mathbf{H}^{H} \mathbf{T}[k]^{H}\right)^{-1}$, we need not explicitly compute the inverse of the matrix $\mathbf{T}[k] \mathbf{H H}^{H} \mathbf{T}[k]^{H}$. In fact $\left(\mathbf{T}[k] \mathbf{H H}^{H} \mathbf{T}[k]^{H}\right)^{-1}$ turns out to be a row and column permuted version of $\left(\mathbf{H H}^{H}\right)^{-1}$. To see this, we note that since $\mathbf{T}[k]$ are permutation matrices, $\mathbf{T}[k]^{H}=\mathbf{T}[k]^{-1}$ and therefore

$$
\left(\mathbf{T}[k] \mathbf{H H}^{H} \mathbf{T}[k]^{H}\right)^{-1}=\mathbf{T}[k]\left(\mathbf{H H}^{H}\right)^{-1} \mathbf{T}[k]^{H} .
$$

To be precise, exactly $g$ rows and $g$ columns of $\left(\mathbf{H H}^{H}\right)^{-1}$ are permuted, and hence the complexity of computing $\mathbf{A}[k]$ from $\left(\mathbf{H} \mathbf{H}^{H}\right)^{-1}$ is $O\left(g N_{u}\right)$. Since for computing $\mathbf{R}[k]$, we are only interested in the upper $g \times g$ sub-matrix of $\mathbf{A}[k]$, it can be concluded that the complexity of computing $\mathbf{R}[k]$ from $\left(\mathbf{H H}^{H}\right)^{-1}$ is only $O\left(g^{3}\right)$ (permuting $\left(\mathbf{H H}^{H}\right)^{-1}$ to get the upper $g \times g$ sub-matrix of $\mathbf{A}[k]$ has a complexity of $O\left(g^{2}\right)$ and that of inverting it is $O\left(g^{3}\right)$ ).

\section{B. Computation of $\mathbf{Q}[k]$ from $\left(\mathbf{H H}^{H}\right)^{-1}$}

In the following we firstly show how $\mathbf{F}[k]$ can be computed efficiently from $\mathbf{A}[k]$ (see (65)). Since $\mathbf{F}[k]=\mathbf{Q}[k] \mathbf{R}[k], \mathbf{Q}[k]$ can then be computed from the QR-decomposition of $\mathbf{F}[k]$.

Right multiplication of $\mathbf{A}[k]$ by the inverse of its upper $g \times g$ sub-matrix gives

$$
\begin{array}{r}
\mathbf{A}[k]\left(\mathbf{G}[k] \mathbf{G}[k]^{H}-\mathbf{G}[k] \mathbf{H}[k]^{H}\left(\mathbf{H}[k] \mathbf{H}[k]^{H}\right)^{-1} \mathbf{H}[k] \mathbf{G}[k]^{H}\right) \\
=\left[\begin{array}{c}
\mathbf{I}_{g} \\
\left.-\left(\mathbf{H}[k] \mathbf{H}[k]^{H}\right)^{-1} \mathbf{H}[k] \mathbf{G}[k]^{H}\right] .(69)
\end{array}\right.
\end{array}
$$

The complexity of computing the inverse of the upper $g \times g$ sub-matrix of $\mathbf{A}[k]$ is $O\left(g^{3}\right)$. The complexity of the right multiplication in (69) is $O\left(g^{2} N_{u}\right)$. Further pre-multiplication with $\mathbf{H}^{H} \mathbf{T}[k]^{H}$ gives the desired matrix $\mathbf{F}[k]$ (see (67)). The complexity of matrix multiplication on the left hand side of (67) is $O\left(g N_{u} N_{t}\right)$. The complexity of computing the QR-decomposition for $\mathbf{F}[k] \in \mathbb{C}^{N_{t} \times g}$ is $O\left(g^{2} N_{t}\right)$. We also know from the previous section that the complexity of computing $\mathbf{A}[k]$ from $\left(\mathbf{H} \mathbf{H}^{H}\right)^{-1}$ is $O\left(g N_{u}\right)$. Summing up the discussion above, it follows that the total complexity of computing $\mathbf{Q}[k]$ from $\left(\mathbf{H H}^{H}\right)^{-1}$ is $O\left(g^{3}\right)+O\left(g^{2} N_{u}\right)+$ $O\left(g N_{u} N_{t}\right)+O\left(g^{2} N_{t}\right)$.

\section{REFERENCES}

[1] I. E. Telatar, "Capacity of multi-antenna Gaussian channels," European Trans. on Telecommunications, pp. 585-595, vol. 10, no. 6, Dec. 1999.

[2] H. Weingarten, Y. Steinberg, S. Shamai, "The capacity region of the Gaussian multiple-input multiple-output broadcast channel," IEEE Trans. on Information Theory, pp. 3936-3964, vol. 52, no. 9, Sept. 2006.

[3] N. Jindal, and A. Goldsmith, "Dirty-paper coding versus TDMA for MIMO broadcast channels," IEEE Trans. on Information Theory, pp. 1783-1794, vol. 51, no. 5, May 2005.

[4] N. Jindal, W. Rhee, S. Vishwanath, S. A. Jafar and A. Goldsmith, "Sum Power Iterative Water-Filling for Multi-Antenna Gaussian Broadcast Channels," IEEE Trans. on Information Theory, pp. 1570-1580, vol. 51, no. 4, April 2005.

[5] C. B. Peel, B. M. Hochwald, and A. L. Swindlehurst, "A vector-perturbation technique for near-capacity multiantenna multiuser communication-part I: channel inversion and regularization," IEEE Trans. on Communications, pp. 195-202, vol. 53, no. 1, Jan. 2005.

[6] C. Windpassinger and R. F. H. Fischer, "Low-complexity near-maximumlikelihood detection and precoding for MIMO systems using lattice reduction," Proc. of Information Theory Workshop (ITW'2003), Paris, France, March 31 - April 4, 2003.

[7] P. W. Baier, M. Meurer, T. Weber, and H. Troeger, "Joint transmission (JT), an alternative rationale for the downlink of Time Division CDMA using multi-element transmit antennas," Proc. of IEEE Int. Symp. on Spread Spectrum Techniques and Applications (ISSSTA'2000), pp. 1-5, New Jersey, USA, Sept. 6-8 2000.

[8] W. Yu, D. P. Varodayan, and J. M. Cioffi, "Trellis and Convolutional Precoding for Transmitter-Based Interference Presubtraction," IEEE Trans. on Communications, pp. 1220-1230 , vol. 53, no. 7, July 2005.

[9] Y. Sun, Y. Yang, A. Liveris, V. Stankovic and Z. Xiong, "Near capacity dirty-paper code design : A source channel coding approach," IEEE. Trans. on Information Theory, pp. 3013-3031, vol. 55, no. 7, July 2009.

[10] G. Shilpa, A. Thangaraj, and S. Bhashyam, "Dirty paper coding using sign-bit shaping and LDPC codes," Proc. IEEE International Symposium on Information Theory (ISIT'2010), pp. 923-927, Austin, Texas, June 13-18 2010.

[11] S. Huang, H. Yin, J. Wu, V. C. M. Leung, "User selection for multiuser MIMO downlink with zero-forcing beamforming," IEEE Trans. on Vehicular Tech. pp., vol. 62, no. 7, Sept. 2013.

[12] O. B. Karimi, M. A. Toutounchain, J. Liu, C. Wang, "Light weight user grouping with flexible degrees of freedom in virtual MIMO," IEEE Journ. on Sel. Areas, in Commun., pp. 2004-2012 vol. 31, no. 10, Oct. 2013. 
[13] Z. Shen, R. Chen, J. G. Andrews, R. W. Heath, and B. L. Evans, "Low complexity user selection algorithms for multiuser MIMO systems with block diagonalization," IEEE Trans. on Signal processing, pp. 3658-3663, vol. 54, no. 9, Sept. 2006.

[14] Z. Tu, and R. Blum, "Multiuser diversity for a dirty paper approach," IEEE Communication Letters, vol. 7, no. 8, pp. 370-372, Aug. 2003.

[15] M. Razaviyayn, M. Baligh, A. Callard, Z. Luo, "Joint user grouping and transceiver design in MIMO interfering broadcast channel," IEEE Trans. on Signal Processing, pp. 85-94, vol. 62, no. 1, Jan. 2014.

[16] Q. H. Spencer, A. L. Swindlehurst, and M. Haardt, "Zero-Forcing methods for downlink spatial multiplexing in multiuser MIMO channels," IEEE Trans. on Signal processing, pp. 461-471, vol. 52, no. 2, Feb. 2004.

[17] R. Chen, C. Li, J. Li and Y. Zhang, "Low complexity user grouping vector perturbation," Wireless Communications Letters, pp. 189-192, vol. 1, no. 3, March 2012.

[18] S. K. Mohammed, and E. G. Larsson, "A low complexity user grouping based multiuser MISO downlink precoder," in Proc. IEEE Global Communications Conference (GLOBECOM’2011), Houston, TX, USA, Dec. 2011.

[19] M. Costa, "Writing on dirty paper," IEEE. Trans. on Information Theory, pp. 439-441, vol. IT-29, May 1983.

[20] G. Caire and S. Shamai, "On the achievable throughput of a multiantenna Gaussian broadcast channel," IEEE. Trans. on Information Theory, pp. 1691-1706, vol. 49, no. 7, July 2003.

[21] U. Erez, S. Shamai and R. Zamir, "Capacity and lattice-strategies for canceling known interference," IEEE. Trans. on Information Theory, pp. 3820-3833, vol. 51, no. 11, Nov. 2005.

[22] T.M. Cover and Joy A. Thomas, Elements of information theory, John Wiley and Sons, 2nd Ed., July 2006.

[23] A. M. Tulino and S. Verdu, "Random matrix theory and wireless communications," Foundation and Trends in Communications and Information Theory, Now Publishers, vol. 1, no. 1, 2004.

[24] M. Abramowitz and I. A. Stegun, "Handbook of Mathematical Functions," National Bureau of Standards, Applied Mathematics Series 55, Ninth printing, Nov. 1970.

[25] S. Vishwanath, N. Jindal, A. Goldsmith, "Duality, achievable rates and sum-rate capacity of Gaussian MIMO broadcast channels," IEEE Trans. on Information Theory, pp. 2658-2668, vol. 49, no. 10, Oct. 2003.

[26] R. A. Horn, and C. R. Johnson, "Matrix Analysis," Cambridge University Press, 1985.

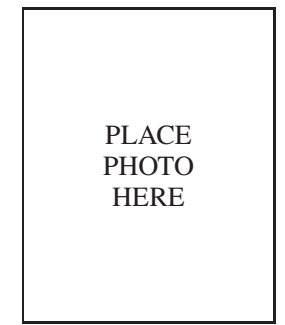

Saif Khan Mohammed (S'08-M'11) Saif Khan Mohammed (S'08-M'11) received the B.Tech degree in Computer Science and Engineering from the Indian Institute of Technology (I.I.T.), New Delhi, India, in 1998 and the Ph.D. degree from the Electrical and Communication Engineering Department, Indian Institute of Science, Bangalore, India, in 2010. Currently he is an Assistant Professor in the Department of Electrical Engineering, I.I.T. Delhi. From Sept. 2011 to Feb. 2013, he was Assistant Professor at the Communication Systems Division (Commsys) in the Electrical Engineering Department (ISY) at Linkoping University, Sweden. From 2010 to 2011, he was a postdoctoral researcher at Commsys.

He has previously worked as a Systems and Algorithm designer in the Wireless Systems Group at Texas Instruments, Bangalore (India) (2003 2007). From 2000 to 2003, he worked with Ishoni Networks, Inc., Santa Clara, CA (USA), as a Senior Chip Architecture Engineer. From 1998 to 2000, he was a ASIC Design Engineer with Philips, Inc., Bangalore. His main research interests include wireless communication using large antenna arrays, coding and signal processing for wireless communication systems, information theory and statistical signal processing. He holds four granted US patents in the area of multi-user detection and precoding for multipleinput multiple-output (MIMO) communication systems.

$\mathrm{He}$ is a member of the IEEE, the IEEE Communication Society, the IEEE Signal Processing Society and the IEEE Information Theory Society. He currently serves as an Editor for the IEEE Wireless Communications Letters. He has also served as a guest editor for the special issue on Massive MIMO in the Journal of Communication Networks (JCN). He has been a Technical Program Committee member for several IEEE conferences (International Conference on Communications (ICC' 2013, 2014, 2015), the IEEE Global Communications Conference (GLOBECOM 2015), the IEEE Vehicular Technology Conference (VTC) Spring 2013, and the IEEE Swedish Communication Theory Workshop (Swe-CTW) Fall 2012). Dr. Mohammed was awarded the Young Indian Researcher Fellowship by the Italian Ministry of University and Research (MIUR) for the year 2009-10. He was also awarded the CENIIT (Linkoping University) research grant for the year 2012.

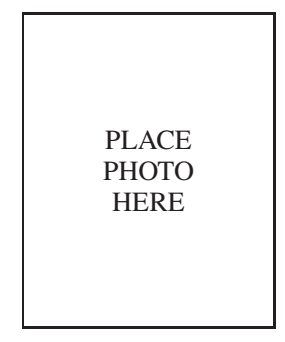

Erik G. Larsson Erik G. Larsson received his Ph.D. degree from Uppsala University, Sweden, in 2002. Since 2007, he is Professor and Head of the Division for Communication Systems in the Department of Electrical Engineering (ISY) at Linköping University (LiU) in Linköping, Sweden. He has previously been Associate Professor (Docent) at the Royal Institute of Technology (KTH) in Stockholm, Sweden, and Assistant Professor at the University of Florida and the George Washington University, USA. In the spring of 2015 he was a Visiting Fellow at Princeton University, USA, for four months.

His main professional interests are within the areas of wireless communications and signal processing. He has published some 100 journal papers on these topics, he is co-author of the textbook Space-Time Block Coding for Wireless Communications (Cambridge Univ. Press, 2003) and he holds 15 issued and many pending patents on wireless technology.

He has served as Associate Editor for several major journals, including the IEEE Transactions on Communications (2010-2014) and IEEE Transactions on Signal Processing (2006-2010). He serves as chair of the IEEE Signal Processing Society SPCOM technical committee in 2015-2016 and as chair of the steering committee for the IEEE Wireless Communications Letters in 2014-2015. He is the General Chair of the Asilomar Conference on Signals, Systems and Computers in 2015 (he was Technical Chair in 2012). He received the IEEE Signal Processing Magazine Best Column Award twice, in 2012 and 2014 , and he is receiving the IEEE ComSoc Stephen O. Rice Prize in Communications Theory in 2015. 